Check for updates

Cite this: RSC Adv., 2017, 7, 30594

Received 29th April 2017

Accepted 8th June 2017

DOI: 10.1039/c7ra04817c

rsc.li/rsc-advances

\section{Regio- and stereoselective syntheses of allylic thioethers under metal free conditions $\uparrow$}

\author{
Pratibha Singh, $\$$ Rekha Bai, $\$$ Rakhee Choudhary, $\$$ Mahesh C. Sharma \\ and Satpal Singh Badsara (D *
}

A metal free, regio and stereoselective syntheses of allylic thioethers using allyl iodides and aryl or alkyl disulfides as coupling partners is described. The densely functionalized allyl iodides having different stereochemistry $(E \& Z)$ reacted well with a variety of disulfides in a regio and stereoselective manner providing the resulting allyl aryl thioethers in $62-92 \%$ yields.

\section{Introduction}

Due to environmental concerns and cost issues, the metal-free organic transformations are in great need. Enough progress has been made in this direction by using various catalytic systems in recent years; ${ }^{1}$ the peroxide alone or with additives has emerged as the perfect substitute to the traditional transition metal catalysis for several organic transformations. ${ }^{2}$ Meanwhile, aryl thioethers have been found to play important roles in organic synthesis, the pharmaceutical industry and materials science. ${ }^{3,4}$ Various transition metals such as $\mathbf{P d},{ }^{5 a-d}$ $\mathrm{Cu},{ }^{5 e-g} \mathrm{Fe},{ }^{5 h, i} \mathrm{Ni},{ }^{5 j, k} \mathrm{In},{ }^{5 l} \mathrm{Co},{ }^{5 m} \mathrm{Au},{ }^{5 n} \mathrm{Ag},{ }^{5 o} \mathrm{Mg}^{5 p}$ etc. have been used so far for the syntheses of thioethers via $\mathrm{C}-\mathrm{S}$ bond formation between aryl halides or pseudo halides and thiols. ${ }^{6}$ Recently, the syntheses of aryl thioethers and thioesters have been reported under metal-free conditions via $\mathrm{C}-\mathrm{H}$ functionalization using a variety of sulphur surrogates. ${ }^{7,8}$ Various catalyst or catalytic systems such as DTBP, ${ }^{7 \boldsymbol{a}, \boldsymbol{c}, \boldsymbol{d}, \boldsymbol{f}, \boldsymbol{g} \text { TBHP }}{ }^{7 \boldsymbol{h}, \boldsymbol{i}} \mathrm{K}_{2} \mathrm{~S}_{2} \mathrm{O}_{8},{ }^{7 j-l}$ AcOOH, ${ }^{8 c}$ etc. have been used so far for the syntheses of thioethers via $\mathrm{C}-\mathrm{H}$ functionalization. In the case of syntheses of allyl aryl thioethers, various metals such as $\mathrm{Rh}^{9 \boldsymbol{a}} \mathrm{In},{ }^{9 b} \mathrm{Co},{ }^{9 c} \mathrm{Ni},{ }^{9 d}$ etc. were used for the $\mathrm{C}-\mathrm{S}$ coupling between allyl halides/acetates and disulfides (Scheme 1). A palladium acetate catalyzed synthesis of allyl aryl thioethers via cross-coupling reaction between Baylis-Hillman acetates and diphenyl disulfides was reported by Sreedhar and co-workers (Scheme 1). ${ }^{p e}$ The syntheses of allyl aryl thioethers via the $\mathrm{C}-\mathrm{S}$ bond formation between allyl halides and sulphur surrogate under metal free conditions is not well studied. Therefore, we have decided to find out suitable methodology for the synthesis of allylic thioethers and herein report the first regio- and stereoselective syntheses of allylic thioethers

MFOS Laboratory, Department of Chemistry (Centre of Advanced Study), University of Rajasthan, JLN Marg, Jaipur, Rajasthan, India-302004.E-mail: badsarass4@uniraj. ac.in; sattubhu2005@gmail.com

$\dagger$ Electronic supplementary information (ESI) available. See DOI: $10.1039 / \mathrm{c} 7 \mathrm{ra} 04817 \mathrm{c}$

$\ddagger$ Authors contributed equally. via $\mathrm{C}-\mathrm{S}$ bond formation between densely functionalized allyl iodides and disulfides under metal free conditions (Scheme 2).

\section{Results and discussion}

Accordingly, we have selected the allyl iodide $1 \mathrm{a}(2.0 \mathrm{mmol})$ and diphenyl disulfide $\mathbf{2 a}(1.0 \mathrm{mmol})$ as model substrate and treated them under the influence of DCP (dicumyl peroxide, $10.0 \mathrm{mmol}$ ) using $\mathrm{CH}_{3} \mathrm{CN}$ as solvent at $80{ }^{\circ} \mathrm{C}$ for $48 \mathrm{~h}$. The data collection of isolated product revealed the formation of only allyl phenyl thioether $4 \mathrm{a}$ in $42 \%$ yield (Table 1 , entry 1 ). There was no formation of the thioethers $\mathbf{3 a}$ and $\mathbf{3} \mathbf{b}$ which were confirmed by GCMS of crude reaction mixture. It is to note here that complete retention in stereochemistry across the double bond was obtained in this reaction i.e. $Z$-selectivity.

Encouraged by these results, we decided to optimize the reaction conditions for this fascinating $\mathrm{C}-\mathrm{S}$ bond formation strategy. Accordingly, a variety of oxidants were employed to catalyze the reaction between $1 \mathrm{a}$ and $2 \mathbf{a}$ (Table 1). Oxidant such as BPO (benzoyl peroxide), $\mathrm{K}_{2} \mathrm{~S}_{2} \mathrm{O}_{8}$ could not provide the encouraging results (entries 2 \& 3). $\mathrm{H}_{2} \mathrm{O}_{2}$, TBHP (tert-butyl

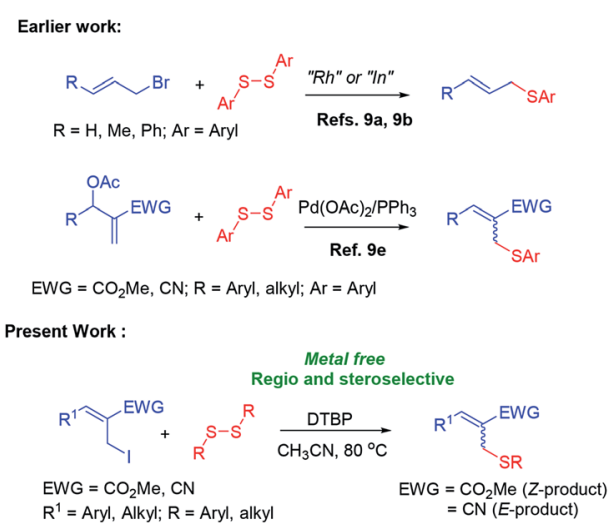

Scheme 1 Syntheses of allylic thioethers. 


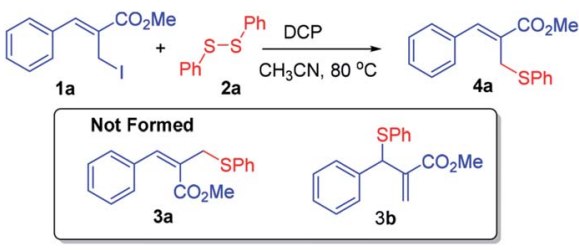

Scheme 2 Synthesis of allyl aryl thioethers $4 a$.

Table 1 Optimization of the reaction conditions ${ }^{a}$

\begin{tabular}{|c|c|c|c|}
\hline Entry & Oxidant (equiv.) & Time (h) & Yield $^{b}(\%$ \\
\hline 1 & DCP $(5.0)$ & 12 & 42 \\
\hline 2 & BPO (5.0) & 12 & 36 \\
\hline 3 & $\mathrm{~K}_{2} \mathrm{~S}_{2} \mathrm{O}_{8}(5.0)$ & 12 & 30 \\
\hline 4 & $\mathrm{H}_{2} \mathrm{O}_{2}(5.0)$ & 12 & 51 \\
\hline $5^{c}$ & TBHP (5.0) & 12 & 58 \\
\hline 6 & ТВРВ (5.0) & 12 & 55 \\
\hline 7 & DTBP (5.0) & 12 & 60 \\
\hline 8 & DTBP (5.0) & 48 & 68 \\
\hline $9^{d}$ & DTBP (5.0) & 48 & 88 \\
\hline $10^{e}$ & DTBP (5.0) & 48 & 82 \\
\hline $11^{f}$ & DTBP (5.0) & 48 & 76 \\
\hline $12^{g}$ & DTBP $(2.0)$ & 48 & 48 \\
\hline
\end{tabular}

${ }^{a}$ Reaction conditions: allyl iodide $\mathbf{1 a}(2.0 \mathrm{mmol})$, diphenyl disulfide $\mathbf{2 a}$ $(1.0 \mathrm{mmol})$ and oxidant $(10.0 \mathrm{mmol})$ were reacted in $\mathrm{CH}_{3} \mathrm{CN}(2.0 \mathrm{~mL})$ at $80{ }^{\circ} \mathrm{C}$ for $12 \mathrm{~h} .{ }^{b}$ Isolated yields are based on 1a. ${ }^{c}$ TBHP solution in water. ${ }^{d} 2.0 \mathrm{mmol}$ of $2 \mathrm{a}$ was used. ${ }^{e} 120{ }^{\circ} \mathrm{C} .{ }^{f} 1.0 \mathrm{~mL} \mathrm{CH} \mathrm{CH}_{3} \mathrm{CN}$ was used. ${ }^{g} 2.0$ equivalent of DTBP was used.

hydroperoxide) and TBPB (tert-butyl peroxybenzoate) provided slightly better results (entries 4-6). The DTBP (di-tert-butyl peroxide) provided the desired product in $60 \%$ yield after $12 \mathrm{~h}$ (entry 7). When the same reaction was carried out for $48 \mathrm{~h}$ under the influence of DTBP, provided the thioether $4 \mathrm{a}$ in $68 \%$ yield (entry 8). Interestingly, when the amount of disulfide was doubled, thioether 4 a was obtained in $88 \%$ yield (entry 9). Enhancement in the reaction temperature could not increase the yield significantly (entry 10). Diminishing the $\mathrm{CH}_{3} \mathrm{CN}$ and DTBP amounts were not found favourable for this coupling between $1 \mathrm{a}$ and $2 \mathrm{a}$ (entry 11 \& 12).

Once we have optimized reaction conditions in hand (Table 1 , entry 9), we then studied the substrate scope for this interesting C-S bond formation. Accordingly, a variety of allyl iodides $\mathbf{1}$ and $\mathbf{5}$ were synthesized from the Baylis-Hillman alcohols following the literature procedures. ${ }^{10 a, b}$ It is worth mentioning here that Baylis-Hillman adducts or their derivatives possessing ester functionality (obtained from alkyl acrylates) and nitrile functionality (obtained from acrylonitrile) be evidence for remarkable opposite stereochemical directions in various organic transformations. ${ }^{\mathbf{1 0 a}, \boldsymbol{b}, \mathbf{1 1}}$ This effect of reversibility might be attributed to the steric difference between the nitrile (smaller) and ester (larger) functionalities. The alcohols possessing ester functionality provided the allyl iodides as $Z$ isomer ${ }^{12}$ only whereas the alcohols possessing nitrile functionality provided allyl iodides as $E$-isomer. Therefore, $Z$-isomer of allyl iodides $\mathbf{1}$ and $E$-isomer of allyl iodide $\mathbf{5}$ was used as coupling partner for $\mathrm{C}-\mathrm{S}$ bond formation with disulfides.

Firstly, the various allyl iodides possessing ester functionality with $Z$ stereochemistry were treated with different disulfides 2 under the influence of DTBP following the optimized reaction conditions, provided the resulting allylic thioethers 4 in $62-85 \%$ isolated yields. The structures of these allylic thioethers 4 were determined from their spectral $\left({ }^{1} \mathrm{H}\right.$ NMR, ${ }^{13} \mathrm{C}$ NMR and MS) data which suggested complete retention in stereochemistry across the double bond in the products i.e. $Z$ stereochemistry were observed (in ${ }^{1} \mathrm{H}$ NMR the olefinic proton appeared at $\delta$ 7.64-7.81 range confirm $Z$-stereochemistry). ${ }^{10 a, b, 11}$ Allyl iodides possessing substitutes at $o / \mathrm{m} / \mathrm{p}$-position underwent regio- and stereo selective C-S coupling reaction with both aryl and alkyl disulfides to provide the desired allyl aryl thioethers (4a-d \& $\mathbf{4} \mathbf{f}-\mathbf{r})$ and allyl alkyl thioethers (4e \& $\mathbf{4 s}-\mathbf{v})$ in good to excellent yields. We have also employed the allyl iodides $\mathbf{1}(E-$ isomer) possessing alkyl group instead that of aryl group for the $\mathrm{C}-\mathrm{S}$ bond formation with disulfides under the optimized reaction conditions, provided the desired allyl aryl thioethers $\mathbf{4 w}$ and $\mathbf{4 x}$ in $72 \%$ and $70 \%$ yields respectively. In these case also complete retention in stereochemistry across the double bond in the products i.e. $E$-stereochemistry were observed..$^{12}$

Subsequently, we have employed the densely functionalized $E$-allyl iodides 5 possessing nitrile functionality for the $\mathrm{C}-\mathrm{S}$ coupling with variety of disulfides 2 under the influence of DTBP following the optimized reaction conditions. Both the aryl and alkyl disulfide coupled well with allyl iodides $\mathbf{5}$ to provide the resulting allylic thioethers 6 in $72-92 \%$ yield. Substrates possessing substituent at $o, m, p$ position of phenyl ring coupled well under the reaction conditions employed. The structures of these allylic thioethers 6 were determined from their spectral $\left({ }^{1} \mathrm{H}\right.$ NMR, ${ }^{13} \mathrm{C}$ NMR and MS) data which suggest that a complete retention in stereochemistry across the double bond in the products i.e. $E$ stereochemistry were observed (in ${ }^{1} \mathrm{H}$ NMR the olefinic proton appeared at $\delta$ 6.47-6.76 range confirm $E$-stereochemistry). ${ }^{10 a, b, 11}$

To establish a possible reaction pathway for this methodology, we have performed few control experiments as shown in Scheme 3. Initially, we have performed the C-S coupling reaction between allyl iodide 1a and disulfide 2a in absence of DTBP

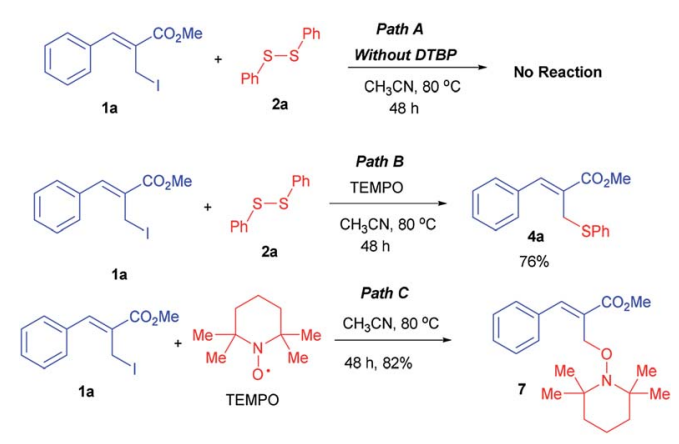

Scheme 3 Control experiments. 


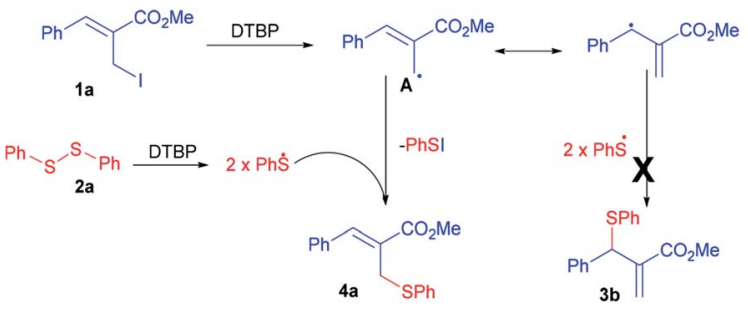

Scheme 4 Plausible mechanism for the synthesis of thioether $4 a$.

under optimized reaction conditions and observed that no reaction took place (Path A, Scheme 3). Next, the same reaction was carried out in presence of TEMPO (2,2,6,6-tetramethylpiperidine- $N$-oxyl) using optimized reaction conditions which provided the allylic thioether $\mathbf{4 a}$ in $76 \%$ isolated yield (Path B, Scheme 3). It was found that the TEMPO coupled well with allyl iodide 1a under same reaction conditions to provide coupled product 7 in $82 \%$ isolated yield (Path C, Scheme 3). On the basis of these control experiments we proposed plausible mechanism which follows the radical pathway (Scheme 4).

A plausible mechanism for the syntheses of allylic thioethers is presented in the Scheme 4 by taking $\mathbf{4 a}$ as model case. In the presence of DTBP, the allyl iodide 1a generated allyl radical A. At the same time disulfide converted into phenyl sulphide radical. The coupling of allyl radical $\mathbf{A}$ with phenyl sulphide radical yielded into the resulting thioether $\mathbf{4 a}$.

\section{Conclusions}

In conclusions, we have developed a methodology for the synthesis of allylic thioethers via $\mathrm{C}-\mathrm{S}$ bond formation between allyl iodides and disulfides under metal free conditions for the first time. A variety of densely functionalized allyl iodides and disulfides coupled well under the influence of DTBP provided the thioethers in $62-92 \%$ yield. A complete stereo- and regioselectivity were observed in these transformations.

\section{Experimental}

\section{General information}

All chemicals were purchased from commercial suppliers and used without further purification. NMR spectra were recorded on a Jeol resonance-400 instrument using $\mathrm{CDCl}_{3}$ as solvent. Chemical shifts are reported in parts per million (ppm) and referenced to the residual solvent resonance. Coupling constant $(J)$ are reported in hertz $(\mathrm{Hz})$. Standard abbreviations indicating multiplicity were used as follows: $\mathrm{s}=$ singlet, $\mathrm{d}=$ doublet, $\mathrm{t}=$ triplet, $\mathrm{dd}=$ double doublet, $\mathrm{q}=$ quartet, $\mathrm{m}=$ multiplet. HRMS data were collected on Waters - Xevo G2S QTof with UPLC HClass Ultra Performance Liquid chromatography-mass spectrometry (LC-MS) facility.

\section{General procedure for Table 1}

To a stirred solution of allyl iodide 1a i.e. methyl-(Z)-2(iodomethyl)-3-phenylacrylate $(2.0 \mathrm{mmol})$ and diphenyl disulfide $(2.0 \mathrm{mmol}, 0.436 \mathrm{~g})$ in $\mathrm{CH}_{3} \mathrm{CN}(2.0 \mathrm{~mL})$ was added oxidant $(10.0 \mathrm{mmol})$ and then the reaction mixture was stirred for $80{ }^{\circ} \mathrm{C}$ under nitrogen atmosphere for $48 \mathrm{~h}$. The solvent was then removed under reduced pressure and the crude product thus obtained was purified by column chromatography (silica gel, $1 \%$ EtOAc in hexanes) to provide the allyl thioether $\mathbf{4 a}$ as pale yellow colour liquid.

\section{Representative example of Table 1: methyl-(Z)-3-phenyl-2- ((phenylthio)methyl)acrylate (entry 9, 4a) ${ }^{10 c}$}

The title compound was prepared following the general procedure for Table 1, using allyl iodide 1a i.e. methyl-(Z)-2(iodomethyl)-3-phenylacrylate $(2.0 \mathrm{mmol}, 0.604 \mathrm{~g})$, diphenyl disulfide (2.0 mmol, $0.436 \mathrm{~g})$, DTBP (10 mmol, $1.46 \mathrm{~g}, 1.8 \mathrm{~mL})$ and $\mathrm{CH}_{3} \mathrm{CN}(2.0 \mathrm{~mL})$, providing $\mathbf{4 a}$ as pale yellow liquid. Yield: $0.499 \mathrm{~g}, 88 \% ;{ }^{1} \mathrm{H}$ NMR $\left(400 \mathrm{MHz}, \mathrm{CDCl}_{3}\right): \delta 3.77(\mathrm{~s}, 3 \mathrm{H}), 4.05(\mathrm{~s}$, 2H), 7.20-7.32 (m, 3H), 7.38-7.42 (m, 7H), $7.79(\mathrm{~s}, 1 \mathrm{H}) ;{ }^{13} \mathrm{C} \mathrm{NMR}$ (100 MHz, $\left.\mathrm{CDCl}_{3}\right): \delta 32.3,52.3,126.8,128.2,128.7,129.0,129.1$, 129.6, 130.7, 134.8, 136.0, 141.6, 167.6.

\section{General procedure for Table 2}

To a stirred solution of allyl iodide $\mathbf{1}(2.0 \mathrm{mmol})$ and disulfide $(2.0 \mathrm{mmol})$ in $\mathrm{CH}_{3} \mathrm{CN}(2.0 \mathrm{~mL})$ was added DTBP $(10.0 \mathrm{mmol})$, then the reaction mixture was stirred for $48 \mathrm{~h}$ at $80{ }^{\circ} \mathrm{C}$ under nitrogen atmosphere. The solvent was then removed under reduced pressure and the crude product thus obtained was purified by column chromatography (silica gel, 1\% EtOAc in hexanes) to provide the allyl thioether 4.

Methyl-(Z)-2-(((4-chlorophenyl)thio)methyl)-3-phenylacrylate (4b). The title compound was prepared following the general procedure for Table 2, using allyl iodide 1a i.e. methyl-(Z)-2(iodomethyl)-3-phenylacrylate $(2.0 \mathrm{mmol}, \quad 0.604 \mathrm{~g})$, bis(4chlorophenyl)disulfide $(2.0 \mathrm{mmol}, 0.574 \mathrm{~g})$, DTBP $(10 \mathrm{mmol}$, $1.46 \mathrm{~g}, 1.8 \mathrm{~mL})$ and $\mathrm{CH}_{3} \mathrm{CN}(2.0 \mathrm{~mL})$, providing $4 \mathbf{b}$ as pale yellow liquid. Yield: $0.522 \mathrm{~g}, 82 \%$; ${ }^{1} \mathrm{H}$ NMR $\left(400 \mathrm{MHz}, \mathrm{CDCl}_{3}\right): \delta 3.77$ (s, $3 \mathrm{H}), 3.98(\mathrm{~s}, 2 \mathrm{H}), 7.11(\mathrm{~d}, J=8.8 \mathrm{~Hz}, 2 \mathrm{H}), 7.21(\mathrm{~d}, J=8.8 \mathrm{~Hz}, 2 \mathrm{H})$, 7.30-7.32 (m, 5H), $7.73(\mathrm{~s}, 1 \mathrm{H}) ;{ }^{13} \mathrm{C}$ NMR $\left(100 \mathrm{MHz}, \mathrm{CDCl}_{3}\right)$ : $\delta 32.5,52.3,128.0,128.7,129.0,129.1,129.4,132.4,132.9,134.3$, 134.7, 141.6, 167.4; HRMS (ESI) exact mass calcd for $\mathrm{C}_{17} \mathrm{H}_{15} \mathrm{ClO}_{2} \mathrm{~S}+\mathrm{K}(\mathrm{M}+\mathrm{K}), 357.0118$; found: 357.0127.

Methyl-(Z)-2-(((4-bromophenyl)thio)methyl)-3-phenylacrylate (4c). The title compound was prepared following the general procedure for Table 2, using allyl iodide 1a i.e. methyl-(Z)-2(iodomethyl)-3-phenylacrylate $(2.0 \mathrm{mmol}, 0.604 \mathrm{~g})$, bis(4bromophenyl)disulfide $(2.0 \mathrm{mmol}, 0.752 \mathrm{~g})$, DTBP $(10 \mathrm{mmol}$, $1.46 \mathrm{~g}, 1.8 \mathrm{~mL})$ and $\mathrm{CH}_{3} \mathrm{CN}(2.0 \mathrm{~mL})$, providing $4 \mathrm{c}$ as pale yellow liquid. Yield: $0.515 \mathrm{~g}, 71 \% ;{ }^{1} \mathrm{H}$ NMR $\left(400 \mathrm{MHz}, \mathrm{CDCl}_{3}\right): \delta 3.80(\mathrm{~s}$, $3 \mathrm{H}), 4.00(\mathrm{~s}, 2 \mathrm{H}), 7.16(\mathrm{~d}, J=8.8 \mathrm{~Hz}, 2 \mathrm{H}), 7.29(\mathrm{~d}, J=8.8 \mathrm{~Hz}, 2 \mathrm{H})$, 7.31-7.33 (m, 5H), $7.75(\mathrm{~s}, 1 \mathrm{H}) ;{ }^{13} \mathrm{C}$ NMR $\left(100 \mathrm{MHz}, \mathrm{CDCl}_{3}\right)$ : $\delta 32.3,52.4,120.9,127.9,128.7,129.1,129.4,131.9,132.5,141.7$, 167.5; HRMS (ESI) exact mass calcd for $\mathrm{C}_{17} \mathrm{H}_{15} \mathrm{BrO}_{2} \mathrm{~S}+\mathrm{Na}(\mathrm{M}+$ Na), 384.9874; found: 384.9881 .

Methyl-(Z)-2-(((3-chlorophenyl)thio)methyl)-3-phenylacrylate (4d). The title compound was prepared following the general procedure for Table 2, using allyl iodide 1a i.e. methyl-(Z)-2(iodomethyl)-3-phenylacrylate $(2.0 \mathrm{mmol}, \quad 0.604 \mathrm{~g})$, bis(3- 
Table 2 DTBP-promoted C-S bond formation between allyl iodides 1 and disulfides $2^{a, b}$

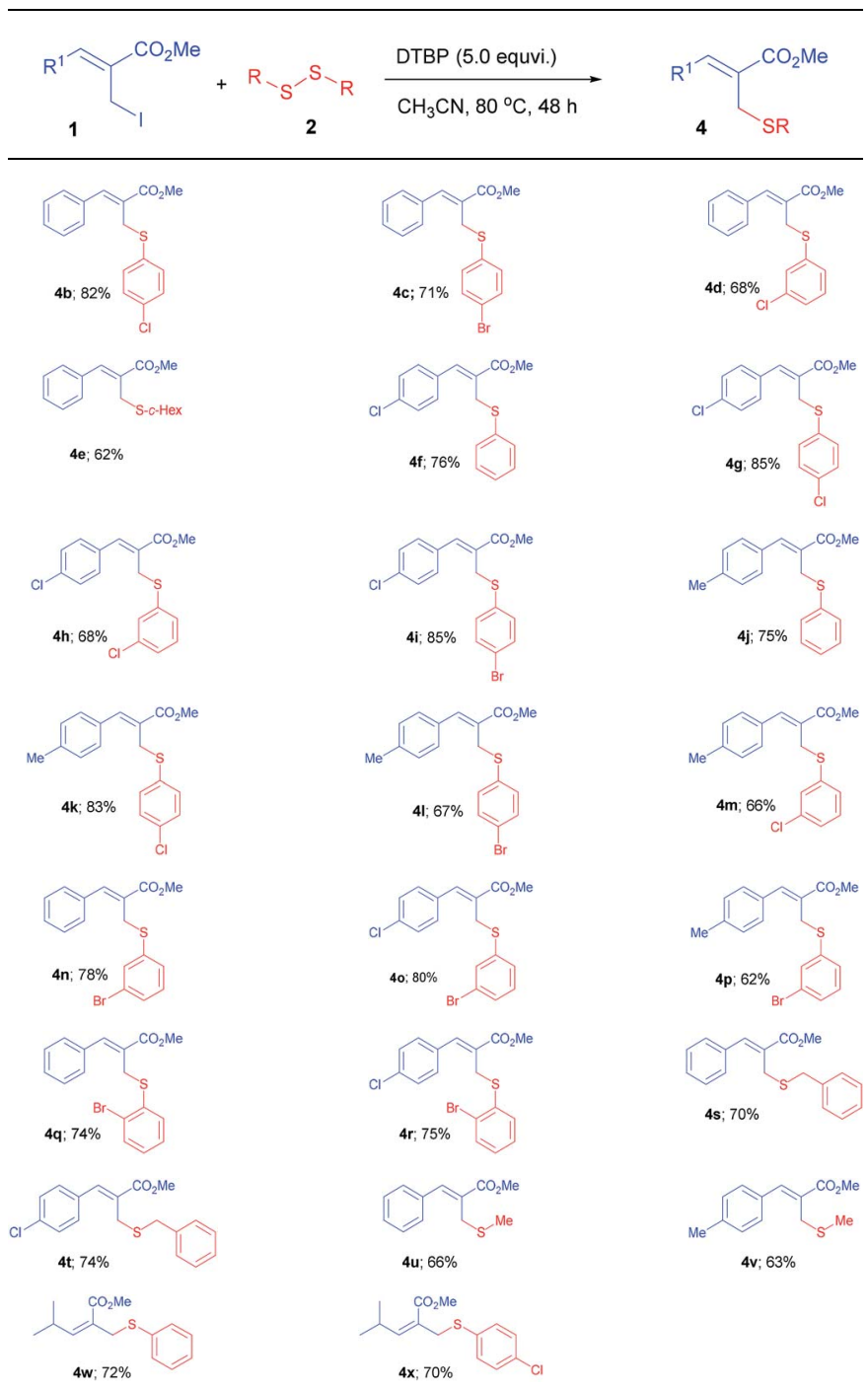

${ }^{a}$ Reaction conditions: allyl iodide $\mathbf{1}(2.0 \mathrm{mmol})$, disulfide $2(2.0 \mathrm{mmol})$ and DTBP $(10.0 \mathrm{mmol})$ were reacted in $\mathrm{CH}_{3} \mathrm{CN}(2.0 \mathrm{~mL})$ at $80^{\circ} \mathrm{C}$ for $48 \mathrm{~h}$. ${ }^{b}$ Isolated yields are based on allyl iodide 1 .

chlorophenyl)disulfide (2.0 mmol, $0.574 \mathrm{~g})$, DTBP $(10 \mathrm{mmol}$, $1.46 \mathrm{~g}, 1.8 \mathrm{~mL})$ and $\mathrm{CH}_{3} \mathrm{CN}(2.0 \mathrm{~mL})$, providing $4 \mathrm{~d}$ as pale yellow liquid. Yield: $0.433 \mathrm{~g}, 68 \% ;{ }^{1} \mathrm{H} \mathrm{NMR}\left(400 \mathrm{MHz}, \mathrm{CDCl}_{3}\right): \delta 3.82(\mathrm{~s}$, $3 \mathrm{H}), 4.05(\mathrm{~s}, 2 \mathrm{H}), 7.08-7.24(\mathrm{~m}, 3 \mathrm{H}), 7.26-7.42(\mathrm{~m}, 6 \mathrm{H}), 7.79(\mathrm{~s}$, $1 \mathrm{H}) ;{ }^{13} \mathrm{C} \mathrm{NMR}\left(100 \mathrm{MHz}, \mathrm{CDCl}_{3}\right): \delta 31.9,52.4,126.7,127.7$, 128.3, 128.8, 129.2, 129.4, 129.8, 129.9, 134.61, 134.65, 138.2, 142.0, 167.5; HRMS (ESI) exact mass calcd for $\mathrm{C}_{17} \mathrm{H}_{15} \mathrm{ClO}_{2} \mathrm{~S}+\mathrm{Na}$ $(\mathrm{M}+\mathrm{Na}), 341.0379$; found: 341.0367.

Methyl-(Z)-2-((cyclohexylthio)methyl)-3-phenylacrylate (4e). The title compound was prepared following the general procedure for Table 2, using allyl iodide 1a i.e. methyl-(Z)-2(iodomethyl)-3-phenylacrylate (2.0 mmol, $0.604 \mathrm{~g})$, dicyclohexyl disulfide (2.0 mmol, $0.44 \mathrm{~mL}$ ), DTBP (10 mmol, $1.46 \mathrm{~g}, 1.8 \mathrm{~mL}$ ) and $\mathrm{CH}_{3} \mathrm{CN}$ (2.0 $\mathrm{mL}$ ), providing $4 \mathrm{e}$ as pale yellow liquid. Yield: $0.359 \mathrm{~g}, 62 \%{ }^{1} \mathrm{H}$ NMR (400 MHz, $\left.\mathrm{CDCl}_{3}\right): \delta 1.06-1.38(\mathrm{~m}, 6 \mathrm{H})$, 1.62-1.75 (m, 2H), 1.78-1.93 (m, 2H), 2.61-2.66 (m, 1H), $3.61(\mathrm{~s}$,
2H), $3.79(\mathrm{~s}, 3 \mathrm{H}), 7.25-7.40(\mathrm{~m}, 3 \mathrm{H}), 7.41-7.52(\mathrm{~m}, 2 \mathrm{H}), 7.68(\mathrm{~s}$, $1 \mathrm{H}) ;{ }^{13} \mathrm{C} \mathrm{NMR}\left(100 \mathrm{MHz}, \mathrm{CDCl}_{3}\right): \delta$ 25.9, 26.1, 26.9, 33.5, 44.5, 52.2, 128.6, 128.9, 129.5, 129.7, 135.1, 140.3, 167.9; HRMS (ESI) exact mass calcd for $\mathrm{C}_{17} \mathrm{H}_{22} \mathrm{O}_{2} \mathrm{~S}+\mathrm{Na}(\mathrm{M}+\mathrm{Na}), 313.1238$; found: 313.1059.

Methyl-(Z)-3-(4-chlorophenyl)-2-((phenylthio)methyl)acrylate (4f). ${ }^{10 c}$ The title compound was prepared following the general procedure for Table 2, using allyl iodide 1b i.e. methyl-(Z)-3-(4chlorophenyl)-2-(iodomethyl)acrylate $(2.0 \mathrm{mmol}, 0.673 \mathrm{~g})$, diphenyl disulfide (2.0 mmol, $0.436 \mathrm{~g})$, DTBP (10 mmol, $1.46 \mathrm{~g}$, $1.8 \mathrm{~mL}$ ) and $\mathrm{CH}_{3} \mathrm{CN}(2.0 \mathrm{~mL})$, providing $\mathbf{4 f}$ as pale yellow liquid. Yield: $0.484 \mathrm{~g}, 76 \% ;{ }^{1} \mathrm{H}$ NMR (400 $\mathrm{MHz}, \mathrm{CDCl}_{3}$ ): $\delta 3.78(\mathrm{~s}, 3 \mathrm{H})$, $3.98(\mathrm{~s}, 2 \mathrm{H}), 7.20-7.282(\mathrm{~m}, 3 \mathrm{H}), 7.34(\mathrm{~s}, 4 \mathrm{H}), 7.36(\mathrm{~d}, J=8.4 \mathrm{~Hz}$, $2 \mathrm{H}), 7.67(\mathrm{~s}, 1 \mathrm{H}) ;{ }^{13} \mathrm{C} \mathrm{NMR}\left(100 \mathrm{MHz}, \mathrm{CDCl}_{3}\right): \delta 32.3,52.4,127.0$, $128.8,128.9$, 129.0, 130.8, 131.1, 133.2, 135.0, 135.6, 140.1, 167.4 .

Methyl-(Z)-3-(4-chlorophenyl)-2-(((4-chlorophenyl)thio)methyl) acrylate (4g). The title compound was prepared following the general procedure for Table 2, using allyl iodide $\mathbf{1 b}$ i.e. methyl(Z)-3-(4-chlorophenyl)-2-(iodomethyl)acrylate $(2.0 \mathrm{mmol}, 0.673$ $\mathrm{g})$, bis(4-chlorophenyl)disulfide (2.0 mmol, $0.574 \mathrm{~g})$, DTBP (10 mmol, $1.46 \mathrm{~g}, 1.8 \mathrm{~mL}$ ) and $\mathrm{CH}_{3} \mathrm{CN}(2.0 \mathrm{~mL})$, providing $4 \mathrm{~g}$ as pale yellow liquid. Yield: $0.600 \mathrm{~g}, 85 \%$; ${ }^{1} \mathrm{H}$ NMR $(400 \mathrm{MHz}$, $\left.\mathrm{CDCl}_{3}\right): \delta 3.77(\mathrm{~s}, 3 \mathrm{H}), 3.93(\mathrm{~s}, 2 \mathrm{H}), 7.14(\mathrm{~d}, J=8.8 \mathrm{~Hz}, 2 \mathrm{H}), 7.222$ $(\mathrm{d}, J=8.8 \mathrm{~Hz}, 2 \mathrm{H}), 7.228(\mathrm{~d}, J=8.8 \mathrm{~Hz}, 2 \mathrm{H}), 7.28(\mathrm{~d}, J=8.8 \mathrm{~Hz}$, $2 \mathrm{H}), 7.64(\mathrm{~s}, 1 \mathrm{H}) ;{ }^{13} \mathrm{C} \mathrm{NMR}\left(100 \mathrm{MHz}, \mathrm{CDCl}_{3}\right): \delta 32.5,52.4,128.5$, 128.9, 129.0, 130.6, 132.6, 133.0, 133.2, 133.9, 135.1, 140.2, 167.2; HRMS (ESI) exact mass calcd for $\mathrm{C}_{17} \mathrm{H}_{14} \mathrm{Cl}_{2} \mathrm{O}_{2} \mathrm{~S}+\mathrm{Na}(\mathrm{M}+$ $\mathrm{Na}), 374.9989$; found: 374.9922 .

Methyl-(Z)-3-(4-chlorophenyl)-2-(((3-chlorophenyl)thio)methyl) acrylate (4h). The title compound was prepared following the general procedure for Table 2, using allyl iodide $\mathbf{1 b}$ i.e. methyl(Z)-3-(4-chlorophenyl)-2-(iodomethyl)acrylate $(2.0 \mathrm{mmol}, 0.673$ g), bis(3-chlorophenyl)disulfide (2.0 mmol, $0.574 \mathrm{~g})$, DTBP (10 mmol, $1.46 \mathrm{~g}, 1.8 \mathrm{~mL})$ and $\mathrm{CH}_{3} \mathrm{CN}(2.0 \mathrm{~mL})$, providing $4 \mathrm{~h}$ as pale yellow liquid. Yield: $0.480 \mathrm{~g}, 68 \% ;{ }^{1} \mathrm{H} \mathrm{NMR}(400 \mathrm{MHz}$, $\left.\mathrm{CDCl}_{3}\right): \delta 3.77(\mathrm{~s}, 3 \mathrm{H}), 3.96(\mathrm{~s}, 2 \mathrm{H}), 7.05-7.20(\mathrm{~m}, 3 \mathrm{H}), 7.22-7.36$ $(\mathrm{m}, 5 \mathrm{H}), 7.66(\mathrm{~s}, 1 \mathrm{H}) ;{ }^{13} \mathrm{C} \mathrm{NMR}\left(100 \mathrm{MHz}, \mathrm{CDCl}_{3}\right): \delta 31.8,52.5$, 126.9 , 128.2, 128.5, 129.0, 129.9, 130.0, 130.7, 133.0, 134.6, 135.2, 137.8, 140.5, 167.1; HRMS (ESI) exact mass calcd for $\mathrm{C}_{17} \mathrm{H}_{14} \mathrm{Cl}_{2} \mathrm{O}_{2} \mathrm{~S}+\mathrm{Na}(\mathrm{M}+\mathrm{Na}), 374.9989$; found: 374.9978 .

Methyl-(Z)-2-(((4-bromophenyl)thio)methyl)-3-(4-

chlorophenyl) acrylate (4i). The title compound was prepared following the general procedure for Table 2, using allyl iodide 1b i.e. methyl-(Z)-3-(4-chlorophenyl)-2-(iodomethyl)acrylate (2.0 mmol, $0.673 \mathrm{~g})$, bis(4-bromophenyl)disulfide $(2.0 \mathrm{mmol}$, $0.752 \mathrm{~g}$ ), DTBP (10 mmol, $1.46 \mathrm{~g}, 1.8 \mathrm{~mL}$ ) and $\mathrm{CH}_{3} \mathrm{CN}(2.0 \mathrm{~mL})$, providing $4 \mathbf{i}$ as yellow solid. Mp: $63{ }^{\circ} \mathrm{C}$; yield: $0.675 \mathrm{~g}, 85 \% ;{ }^{1} \mathrm{H}$ NMR (400 MHz, $\left.\mathrm{CDCl}_{3}\right): \delta 3.77(\mathrm{~s}, 3 \mathrm{H}), 3.93(\mathrm{~s}, 2 \mathrm{H}), 7.14(\mathrm{~d}, J=$ $8.4 \mathrm{~Hz}, 2 \mathrm{H}), 7.22$ (d, $J=8.4 \mathrm{~Hz}, 2 \mathrm{H}), 7.27-7.29(\mathrm{~m}, 4 \mathrm{H}), 7.64(\mathrm{~s}$, $1 \mathrm{H}) ;{ }^{13} \mathrm{C}$ NMR $\left(100 \mathrm{MHz}, \mathrm{CDCl}_{3}\right): \delta 32.3,52.5,121.2,128.4$, 128.9, 130.6, 131.9, 132.7, 133.0, 134.6, 135.1, 140.2, 167.2; HRMS (ESI) exact mass calcd for $\mathrm{C}_{17} \mathrm{H}_{14} \mathrm{BrClO}_{2} \mathrm{~S}+\mathrm{K}(\mathrm{M}+\mathrm{K})$, 434.9223; found: 434.9227.

Methyl-(Z)-2-((phenylthio)methyl)-3-(p-tolyl)acrylate (4j). The title compound was prepared following the general procedure for Table 2, using allyl iodide 1c i.e. methyl-(Z)-2-(iodomethyl)-3- 
(p-tolyl)acrylate $(2.0 \mathrm{mmol}, 0.632 \mathrm{~g})$, diphenyl disulfide (2.0 mmol, $0.436 \mathrm{~g})$, DTBP (10 mmol, $1.46 \mathrm{~g}, 1.8 \mathrm{~mL}$ ) and $\mathrm{CH}_{3} \mathrm{CN}$ $(2.0 \mathrm{~mL})$, providing $\mathbf{4 j}$ as pale yellow liquid. Yield: $0.447 \mathrm{~g}, 75 \%$; ${ }^{1} \mathrm{H}$ NMR (400 MHz, $\left.\mathrm{CDCl}_{3}\right): \delta 2.37(\mathrm{~s}, 3 \mathrm{H}), 3.81(\mathrm{~s}, 3 \mathrm{H}), 4.09(\mathrm{~s}$, $2 \mathrm{H}), 7.18(\mathrm{~d}, J=8.4 \mathrm{~Hz}, 2 \mathrm{H}), 7.21-7.29(\mathrm{~m}, 3 \mathrm{H}), 7.38(\mathrm{~d}, J=$ $8.4 \mathrm{~Hz}, 2 \mathrm{H}), 7.40-7.42(\mathrm{~m}, 2 \mathrm{H}), 7.80(\mathrm{~s}, 1 \mathrm{H}) ;{ }^{13} \mathrm{C} \mathrm{NMR}(100 \mathrm{MHz}$, $\left.\mathrm{CDCl}_{3}\right): \delta 21.5,32.3,52.3,126.7,127.2,129.0,129.5,129.8$, 130.6, 132.0, 136.3, 139.4, 141.9, 167.8; HRMS (ESI) exact mass calcd for $\mathrm{C}_{18} \mathrm{H}_{18} \mathrm{O}_{2} \mathrm{~S}+\mathrm{K}(\mathrm{M}+\mathrm{K}), 337.0665$; found: 337.0652 .

Methyl-(Z)-2-(((4-chlorophenyl)thio)methyl)-3-(p-tolyl)acrylate (4k). The title compound was prepared following the general procedure for Table 2, using allyl iodide 1c i.e. methyl-(Z)-2(iodomethyl)-3-(p-tolyl)acrylate $(2.0 \mathrm{mmol}, 0.632 \mathrm{~g}), \quad$ bis(4chlorophenyl)disulfide $(2.0 \mathrm{mmol}, 0.574 \mathrm{~g})$, DTBP $(10 \mathrm{mmol}$, $1.46 \mathrm{~g}, 1.8 \mathrm{~mL}$ ) and $\mathrm{CH}_{3} \mathrm{CN}(2.0 \mathrm{~mL})$, providing $4 \mathbf{k}$ as pale yellow liquid. Yield: $0.551 \mathrm{~g}, 83 \% ;{ }^{1} \mathrm{H}$ NMR (400 $\left.\mathrm{MHz} \mathrm{CDCl}_{3}\right): \delta 2.35$ (s, $3 \mathrm{H}), 3.79(\mathrm{~s}, 3 \mathrm{H}), 4.03(\mathrm{~s}, 2 \mathrm{H}), 7.15(\mathrm{~d}, J=8.4 \mathrm{~Hz}, 2 \mathrm{H}), 7.16(\mathrm{~d}, J=$ $8.4 \mathrm{~Hz}, 2 \mathrm{H}), 7.26$ (d, $J=8.8 \mathrm{~Hz}, 2 \mathrm{H}), 7.29$ (d, $J=8.8 \mathrm{~Hz}, 2 \mathrm{H}), 7.75$ $(\mathrm{s}, 1 \mathrm{H}) ;{ }^{13} \mathrm{C}$ NMR $\left(100 \mathrm{MHz}, \mathrm{CDCl}_{3}\right): \delta$ 21.4, 32.5, 52.2, 127.0, 128.9, 129.4, 129.6, 131.8, 132.2, 132.9, 134.5, 139.4, 141.9, 167.5; HRMS (ESI) exact mass calcd for $\mathrm{C}_{18} \mathrm{H}_{17} \mathrm{ClO}_{2} \mathrm{~S}+\mathrm{K}(\mathrm{M}+\mathrm{K})$, 371.0275; found: 371.0271 .

Methyl-(Z)-2-(((4-bromophenyl)thio)methyl)-3-(p-tolyl)acrylate (41). The title compound was prepared following the general procedure for Table 2, using allyl iodide 1c i.e. methyl-(Z)-2(iodomethyl)-3-(p-tolyl)acrylate $(2.0 \mathrm{mmol}, 0.632 \mathrm{~g}), \quad$ bis $(4-$ bromophenyl)disulfide (2.0 mmol, $0.752 \mathrm{~g})$, DTBP $(10 \mathrm{mmol}$, $1.46 \mathrm{~g}, 1.8 \mathrm{~mL})$ and $\mathrm{CH}_{3} \mathrm{CN}(2.0 \mathrm{~mL})$, providing 41 as yellow solid. Mp: $61{ }^{\circ} \mathrm{C}$; yield: $0.505 \mathrm{~g}, 67 \% ;{ }^{1} \mathrm{H}$ NMR $\left(400 \mathrm{MHz}, \mathrm{CDCl}_{3}\right): \delta 2.35$ (s, 3H), 3.79 (s, 3H), 4.02 (s, 2H), 7.14-7.19 (m, 4H), 7.27-7.31 $(\mathrm{m}, 4 \mathrm{H}), 7.74(\mathrm{~s}, 1 \mathrm{H}) ;{ }^{13} \mathrm{C}$ NMR $\left(100 \mathrm{MHz}, \mathrm{CDCl}_{3}\right): \delta 21.5,32.3$, 52.3, 120.8, 126.9, 129.5, 129.6, 131.8, 131.9, 132.3, 135.2, 139.5, 142.0, 167.7; HRMS (ESI) exact mass calcd for $\mathrm{C}_{18} \mathrm{H}_{17} \mathrm{BrO}_{2} \mathrm{~S}+\mathrm{Na}$ $(\mathrm{M}+\mathrm{Na}), 399.0030$; found: 398.9935.

Methyl-(Z)-2-(((3-chlorophenyl)thio)methyl)-3-(p-tolyl)acrylate $(\mathbf{4 m})$. The title compound was prepared following the general procedure for Table 2, using allyl iodide 1c i.e. methyl$(Z)$-2-(iodomethyl)-3-(p-tolyl)acrylate $(2.0 \mathrm{mmol}, 0.632 \mathrm{~g})$, bis(3chlorophenyl)disulfide (2.0 mmol, $0.574 \mathrm{~g})$, DTBP $(10 \mathrm{mmol}$, $1.46 \mathrm{~g}, 1.8 \mathrm{~mL})$ and $\mathrm{CH}_{3} \mathrm{CN}(2.0 \mathrm{~mL})$, providing $4 \mathrm{~m}$ as pale yellow liquid. Yield: $0.438 \mathrm{~g}, 66 \% ;{ }^{1} \mathrm{H}$ NMR (400 $\mathrm{MHz}, \mathrm{CDCl}_{3}$ ): $\delta 2.35(\mathrm{~s}, 3 \mathrm{H}), 3.81(\mathrm{~s}, 3 \mathrm{H}), 4.07$ (s, 2H), 7.05-7.24 (m, 5H), 7.25$7.40(\mathrm{~m}, 3 \mathrm{H}), 7.78(\mathrm{~s}, 1 \mathrm{H}) ;{ }^{13} \mathrm{C}$ NMR $\left(100 \mathrm{MHz}, \mathrm{CDCl}_{3}\right): \delta 21.5$, 31.9 , 52.3, 126.6, 128.1, 129.60, 129.65, 129.67, 129.9, 131.8, 134.6, 138.4, 139.6, 142.2, 167.5; HRMS (ESI) exact mass calcd for $\mathrm{C}_{18} \mathrm{H}_{17} \mathrm{ClO}_{2} \mathrm{~S}+\mathrm{Na}(\mathrm{M}+\mathrm{Na}), 355.0535$; found: 355.0531 .

Methyl-(Z)-2-(((3-bromophenyl)thio)methyl)-3-

phenylacrylate (4n). The title compound was prepared following the general procedure for Table 2, using allyl iodide 1a i.e. methyl-(Z)-2-(iodomethyl)-3-phenylacrylate (2.0 mmol, $0.604 \mathrm{~g}$ ), bis(3-bromophenyl)disulfide $(2.0 \mathrm{mmol}, \quad 0.752 \mathrm{~g}), \quad$ DTBP (10 mmol, $1.46 \mathrm{~g}, 1.8 \mathrm{~mL}$ ) and $\mathrm{CH}_{3} \mathrm{CN}(2.0 \mathrm{~mL})$, providing $4 \mathrm{n}$ as pale yellow liquid. Yield: $0.566,78 \% ;{ }^{1} \mathrm{H}$ NMR $(400 \mathrm{MHz}$, $\left.\mathrm{CDCl}_{3}\right): \delta 3.81(\mathrm{~s}, 3 \mathrm{H}), 4.04(\mathrm{~s}, 2 \mathrm{H}), 7.07(\mathrm{t}, J=8.0 \mathrm{~Hz}, 1 \mathrm{H}), 7.23$ $(\mathrm{d}, J=8.0 \mathrm{~Hz}, 1 \mathrm{H}), 7.29(\mathrm{~d}, J=8.0 \mathrm{~Hz}, 1 \mathrm{H}), 7.33-7.44(\mathrm{~m}, 5 \mathrm{H})$, 7.46 (s, $1 \mathrm{H}), 7.79$ (s, $1 \mathrm{H}) ;{ }^{13} \mathrm{C}$ NMR (100 MHz, $\left.\mathrm{CDCl}_{3}\right): \delta 32.0$, 52.4, 122.7, 127.7, 128.8, 128.9, 129.2, 129.4, 129.7, 130.2, 132.7,
134.6, 138.4, 142.0, 167.5; HRMS (ESI) exact mass calcd for $\mathrm{C}_{17} \mathrm{H}_{15} \mathrm{BrO}_{2} \mathrm{~S}+\mathrm{Na}(\mathrm{M}+\mathrm{Na}), 384.9874$; found: 384.9698 .

Methyl-(Z)-2-(((3-bromophenyl)thio)methyl)-3-(4-chlorophenyl) acrylate (4o). The title compound was prepared following the general procedure for Table 2, using allyl iodide $\mathbf{1 b}$ i.e. methyl(Z)-3-(4-chlorophenyl)-2-(iodomethyl)acrylate $(2.0 \mathrm{mmol}, 0.673$ g), bis(3-bromophenyl)disulfide $(2.0 \mathrm{mmol}, 0.752 \mathrm{~g})$, DTBP (10 mmol, $1.46 \mathrm{~g}, 1.8 \mathrm{~mL})$ and $\mathrm{CH}_{3} \mathrm{CN}(2.0 \mathrm{~mL})$, providing 40 as pale yellow liquid. Yield: $0.635 \mathrm{~g}, 80 \%$; ${ }^{1} \mathrm{H}$ NMR $(400 \mathrm{MHz}$, $\left.\mathrm{CDCl}_{3}\right): \delta 3.80(\mathrm{~s}, 3 \mathrm{H}), 3.98(\mathrm{~s}, 2 \mathrm{H}), 7.07(\mathrm{t}, J=8.0 \mathrm{~Hz}, 1 \mathrm{H}), 7.21-$ $7.23(\mathrm{~m}, 1 \mathrm{H}), 7.26-7.32(\mathrm{~m}, 5 \mathrm{H}), 7.43(\mathrm{t}, J=1.6 \mathrm{~Hz}, 1 \mathrm{H}), 7.69(\mathrm{~s}$, $1 \mathrm{H}) ;{ }^{13} \mathrm{C} \mathrm{NMR}\left(100 \mathrm{MHz}, \mathrm{CDCl}_{3}\right): \delta 32.0,52.5,122.7,128.3$, $129.0,129.2$, 129.9, 130.2, 130.7, 133.0, 135.2, 138.0, 140.5, 167.2; HRMS (ESI) exact mass calcd for $\mathrm{C}_{17} \mathrm{H}_{14} \mathrm{BrClO}_{2} \mathrm{~S}+\mathrm{K}(\mathrm{M}+$ K), 434.9223; found: 434.9226.

Methyl-(Z)-2-(((3-bromophenyl)thio)methyl)-3-( $p$-tolyl)acrylate (4p). The title compound was prepared following the general procedure for Table 2, using allyl iodide 1c i.e. methyl-(Z)-2(iodomethyl)-3-(p-tolyl)acrylate $(2.0 \mathrm{mmol}, 0.632 \mathrm{~g})$, bis(3bromophenyl)disulfide $(2.0 \mathrm{mmol}, 0.752 \mathrm{~g})$, DTBP $(10 \mathrm{mmol}$, $1.46 \mathrm{~g}, 1.8 \mathrm{~mL})$ and $\mathrm{CH}_{3} \mathrm{CN}(2.0 \mathrm{~mL})$, providing $4 \mathbf{p}$ as pale yellow liquid. Yield: $0.467 \mathrm{~g}, 62 \% ;{ }^{1} \mathrm{H}$ NMR (400 MHz, $\left.\mathrm{CDCl}_{3}\right): \delta 2.36(\mathrm{~s}$, $3 \mathrm{H}), 3.81(\mathrm{~s}, 3 \mathrm{H}), 4.06(\mathrm{~s}, 2 \mathrm{H}), 7.08(\mathrm{t}, J=8.0 \mathrm{~Hz}, 1 \mathrm{H}), 7.17(\mathrm{~d}, J=$ $8.0 \mathrm{~Hz}, 2 \mathrm{H}), 7.24-7.32(\mathrm{~m}, 4 \mathrm{H}), 7.46(\mathrm{t}, J=1.6 \mathrm{~Hz}, 1 \mathrm{H}), 7.77(\mathrm{~s}$, $1 \mathrm{H}) ;{ }^{13} \mathrm{C} \mathrm{NMR}\left(100 \mathrm{MHz}, \mathrm{CDCl}_{3}\right): \delta 21.5,32.0,52.4,122.7,126.6$, $128.7,129.5$, 129.6, 130.1, 131.7, 132.5, 138.6, 139.6, 142.2, 167.6; HRMS (ESI) exact mass calcd for $\mathrm{C}_{18} \mathrm{H}_{17} \mathrm{BrO}_{2} \mathrm{~S}+\mathrm{Na}(\mathrm{M}+$ $\mathrm{Na}), 399.0030$; found: 399.6734.

Methyl-(Z)-2-(((2-bromophenyl)thio)methyl)-3-phenylacrylate (4q). The title compound was prepared following the general procedure for Table 2, using allyl iodide 1a i.e. methyl-(Z)-2(iodomethyl)-3-phenylacrylate $(2.0 \mathrm{mmol}, 0.604 \mathrm{~g})$, bis(2bromophenyl)disulfide $(2.0 \mathrm{mmol}, 0.752 \mathrm{~g})$, DTBP $(10 \mathrm{mmol}$, $1.46 \mathrm{~g}, 1.8 \mathrm{~mL})$ and $\mathrm{CH}_{3} \mathrm{CN}(2.0 \mathrm{~mL})$, providing $4 \mathrm{q}$ as pale yellow liquid. Yield: $0.537 \mathrm{~g}, 74 \% ;{ }^{1} \mathrm{H} \mathrm{NMR}\left(400 \mathrm{MHz}, \mathrm{CDCl}_{3}\right): \delta 3.81(\mathrm{~s}$, $3 \mathrm{H}), 4.04(\mathrm{~s}, 2 \mathrm{H}), 7.02(\mathrm{td}, J=8.0 \mathrm{~Hz} \& 1.6 \mathrm{~Hz}, 1 \mathrm{H}), 7.19(\mathrm{td}, J=$ $8.4 \mathrm{~Hz} \& 1.2 \mathrm{~Hz}, 1 \mathrm{H}), 7.27$ (dd, $J=6.4 \mathrm{~Hz} \& 1.6 \mathrm{~Hz}, 1 \mathrm{H}), 7.32-7.38$ $(\mathrm{m}, 3 \mathrm{H}), 7.41-7.45(\mathrm{~m}, 2 \mathrm{H}), 7.50(\mathrm{dd}, J=6.4 \mathrm{~Hz} \& 1.6 \mathrm{~Hz}, 1 \mathrm{H})$, $7.81(\mathrm{~s}, 1 \mathrm{H}) ;{ }^{13} \mathrm{C} \mathrm{NMR}\left(100 \mathrm{MHz}, \mathrm{CDCl}_{3}\right): \delta 31.4,52.4,125.1$, 127.2 , 127.6, 127.8, 128.8, 129.2, 129.5, 130.6, 133.0, 134.6, 137.3, 142.4, 167.5; HRMS (ESI) exact mass calcd for $\mathrm{C}_{17} \mathrm{H}_{15} \mathrm{BrO}_{2} \mathrm{~S}+\mathrm{K}(\mathrm{M}+\mathrm{K})$, 400.9613; found: 400.9681 .

Methyl-(Z)-2-(((2-bromophenyl)thio)methyl)-3-(4-chlorophenyl) acrylate (4r). The title compound was prepared following the general procedure for Table 2, using allyl iodide $\mathbf{1 b}$ i.e. methyl(Z)-3-(4-chlorophenyl)-2-(iodomethyl)acrylate $(2.0 \mathrm{mmol}, 0.673$ $\mathrm{g})$, bis(2-bromophenyl)disulfide $(2.0 \mathrm{mmol}, 0.752 \mathrm{~g})$, DTBP (10 mmol, $1.46 \mathrm{~g}, 1.8 \mathrm{~mL}$ ) and $\mathrm{CH}_{3} \mathrm{CN}(2.0 \mathrm{~mL})$, providing $4 \mathrm{p}$ as yellow solid. Mp: $74{ }^{\circ} \mathrm{C}$; yield: $0.595 \mathrm{~g}, 75 \%$; ${ }^{1} \mathrm{H}$ NMR $(400 \mathrm{MHz}$, $\left.\mathrm{CDCl}_{3}\right): \delta 3.80(\mathrm{~s}, 3 \mathrm{H}), 3.99(\mathrm{~s}, 2 \mathrm{H}), 7.03(\mathrm{t}, J=7.2 \mathrm{~Hz}, 1 \mathrm{H}), 7.19(\mathrm{t}$, $J=7.6 \mathrm{~Hz}, 1 \mathrm{H}), 7.26-7.38(\mathrm{~m}, 5 \mathrm{H}), 7.50(\mathrm{~d}, J=8.0 \mathrm{~Hz}, 1 \mathrm{H}), 7.72$ $(\mathrm{s}, 1 \mathrm{H}) ;{ }^{13} \mathrm{C} \mathrm{NMR}\left(100 \mathrm{MHz}, \mathrm{CDCl}_{3}\right): \delta 31.4,52.5,125.4,127.7$, 127.922, 127.928, 129.03, 130.9, 131.0, 133.0, 133.1, 135.2, 136.9, 141.0, 167.2; HRMS (ESI) exact mass calcd for $\mathrm{C}_{17} \mathrm{H}_{14}{ }^{-}$ $\mathrm{BrClO}_{2} \mathrm{~S}+\mathrm{K}(\mathrm{M}+\mathrm{K})$, 434.9223; found: 434.9214.

Methyl-(Z)-2-((benzylthio)methyl)-3-phenylacrylate (4s). The title compound was prepared following the general procedure 
for Table 2, using allyl iodide 1a i.e. methyl-(Z)-2-(iodomethyl)-3phenylacrylate $(2.0 \mathrm{mmol}, 0.604 \mathrm{~g})$, dibenzyl disulfide (2.0 mmol, $0.492 \mathrm{~g}$ ), DTBP (10 mmol, $1.46 \mathrm{~g}, 1.8 \mathrm{~mL}$ ) and $\mathrm{CH}_{3} \mathrm{CN}$ $(2.0 \mathrm{~mL})$, providing $4 \mathrm{~s}$ as pale yellow liquid. Yield: $0.417 \mathrm{~g}, 70 \%$; ${ }^{1} \mathrm{H}$ NMR (400 MHz, $\left.\mathrm{CDCl}_{3}\right): \delta 3.46(\mathrm{~s}, 2 \mathrm{H}), 3.64(\mathrm{~s}, 2 \mathrm{H}), 3.72(\mathrm{~s}$, $3 \mathrm{H}), 7.10-7.16(\mathrm{~m}, 5 \mathrm{H}), 7.21-7.22(\mathrm{~m}, 3 \mathrm{H}), 7.28-7.30(\mathrm{~m}, 2 \mathrm{H})$, $7.63(\mathrm{~s}, 1 \mathrm{H}) ;{ }^{13} \mathrm{C} \mathrm{NMR}\left(100 \mathrm{MHz}, \mathrm{CDCl}_{3}\right): \delta 28.5,37.4,52.3,127.0$, 128.5, 128.7, 129.0, 129.1, 129.8, 134.9, 138.3, 140.8, 168.0; HRMS (ESI) exact mass calcd for $\mathrm{C}_{18} \mathrm{H}_{18} \mathrm{O}_{2} \mathrm{~S}+\mathrm{Na}(\mathrm{M}+\mathrm{Na})$, 321.0925; found: 321.0920.

Methyl-(Z)-2-((benzylthio)methyl)-3-(4-chlorophenyl)acrylate (4t). The title compound was prepared following the general procedure for Table 2, using allyl iodide $\mathbf{1 b}$ i.e. methyl-(Z)-3-(4chlorophenyl)-2-(iodomethyl)acrylate $(2.0 \mathrm{mmol}, 0.673 \mathrm{~g})$, dibenzyl disulfide (2.0 mmol, $0.492 \mathrm{~g}$ ), DTBP (10 mmol, $1.46 \mathrm{~g}$, $1.8 \mathrm{~mL})$ and $\mathrm{CH}_{3} \mathrm{CN}(2.0 \mathrm{~mL})$, providing $4 \mathbf{t}$ as pale yellow liquid. Yield: $0.493 \mathrm{~g}, 74 \% ;{ }^{1} \mathrm{H}$ NMR (400 MHz, $\left.\mathrm{CDCl}_{3}\right): \delta 3.52(\mathrm{~s}, 2 \mathrm{H})$, $3.74(\mathrm{~s}, 2 \mathrm{H}), 3.82(\mathrm{~s}, 3 \mathrm{H}), 7.22-7.32(\mathrm{~m}, 9 \mathrm{H}), 7.65(\mathrm{~s}, 1 \mathrm{H}) ;{ }^{13} \mathrm{C}$ NMR (100 MHz, $\left.\mathrm{CDCl}_{3}\right): \delta 28.3,37.4,52.4,127.1,128.5,128.94$, 128.99, 129.0, 129.5, 131.0, 133.2, 135.0, 138.1, 139.5, 167.7; HRMS (ESI) exact mass calcd for $\mathrm{C}_{18} \mathrm{H}_{17} \mathrm{ClO}_{2} \mathrm{~S}+\mathrm{Na}(\mathrm{M}+\mathrm{Na})$, 355.0535; found: 355.0529 .

Methyl-(Z)-2-((methylthio)methyl)-3-phenylacrylate $\quad(4 \mathbf{u}){ }^{13}$ The title compound was prepared following the general procedure for Table 2, using allyl iodide 1a i.e. methyl-(Z)-2(iodomethyl)-3-phenylacrylate $(2.0 \mathrm{mmol}, 0.604 \mathrm{~g})$, dimethyl disulfide (2.0 mmol, $0.188 \mathrm{~g}$ ), DTBP (10 mmol, $1.46 \mathrm{~g}, 1.8 \mathrm{~mL}$ ) and $\mathrm{CH}_{3} \mathrm{CN}(2.0 \mathrm{~mL})$, providing $\mathbf{4 u}$ as colourless oil. Yield: $0.293 \mathrm{~g}, 66 \% ;{ }^{1} \mathrm{H}$ NMR $\left(400 \mathrm{MHz}, \mathrm{CDCl}_{3}\right): \delta 2.01(\mathrm{~s}, 3 \mathrm{H}), 3.55(\mathrm{~s}$, $2 \mathrm{H}), 3.76(\mathrm{~s}, 3 \mathrm{H}), 7.25-7.34(\mathrm{~m}, 3 \mathrm{H}), 7.40(\mathrm{~d}, J=7.6 \mathrm{~Hz}, 2 \mathrm{H}), 7.68$ $(\mathrm{s}, 1 \mathrm{H}) ;{ }^{13} \mathrm{C} \mathrm{NMR}\left(100 \mathrm{MHz}, \mathrm{CDCl}_{3}\right): \delta 16.1,30.4,52.2,128.6$, 128.8, 129.3, 129.5, 134.9, 140.6, 167.9.

Methyl-(Z)-2-((methylthio)methyl)-3-(p-tolyl)acrylate

$(4 \mathbf{v})$. The title compound was prepared following the general procedure for Table 2, using allyl iodide 1c i.e. methyl-(Z)-2-(iodomethyl)-3-( -tolyl)acrylate $(2.0 \mathrm{mmol}, \quad 0.632 \mathrm{~g})$, dimethyl disulfide (2.0 mmol, $0.188 \mathrm{~g}$ ), DTBP (10 mmol, $1.46 \mathrm{~g}, 1.8 \mathrm{~mL}$ ) and $\mathrm{CH}_{3} \mathrm{CN}(2.0 \mathrm{~mL})$, providing $4 \mathbf{v}$ as colourless oil. Yield: $0.297 \mathrm{~g}, 63 \% ;{ }^{1} \mathrm{H}$ NMR (400 MHz, $\mathrm{CDCl}_{3}$ ): $\delta 2.09$ (s, 3H), 2.35 (s, $3 \mathrm{H}), 3.63(\mathrm{~s}, 2 \mathrm{H}), 3.82(\mathrm{~s}, 3 \mathrm{H}), 7.19(\mathrm{~d}, J=8.0 \mathrm{~Hz}, 2 \mathrm{H}), 7.38(\mathrm{~d}, J=$ $8.0 \mathrm{~Hz}, 2 \mathrm{H}), 7.72(\mathrm{~s}, 1 \mathrm{H}) ;{ }^{13} \mathrm{C}$ NMR (100 MHz, $\left.\mathrm{CDCl}_{3}\right): \delta 16.1$, 21.3, 30.5, 52.1, 128.3, 129.3, 129.6, 132.0, 139.0, 140.7, 168.0; HRMS (ESI) exact mass calcd for $\mathrm{C}_{13} \mathrm{H}_{16} \mathrm{O}_{2} \mathrm{~S}+\mathrm{K}(\mathrm{M}+\mathrm{K})$, 275.0508; found: 275.0501.

Methyl-(E)-4-methyl-2-((phenylthio)methyl)pent-2-enoate (4w). The title compound was prepared following the general procedure for Table 2, using allyl iodide 1d i.e. methyl-(E)-2(iodomethyl)-4-methylpent-2-enoate (2.0 mmol, $0.536 \mathrm{~g})$, diphenyl disulfide (2.0 mmol, $0.436 \mathrm{~g}$ ), DTBP (10 mmol, $1.46 \mathrm{~g}, 1.8 \mathrm{~mL}$ ) and $\mathrm{CH}_{3} \mathrm{CN}(2.0 \mathrm{~mL})$, providing $4 \mathbf{w}$ as pale yellow liquid. Yield: $0.360 \mathrm{~g}, 72 \% ;{ }^{1} \mathrm{H}$ NMR (400 MHz, $\mathrm{CDCl}_{3}$ ): $\delta 0.84(\mathrm{~s}, 3 \mathrm{H}), 0.85$ (s, $3 \mathrm{H}), 2.39-2.45(\mathrm{~m}, 1 \mathrm{H}), 3.71(\mathrm{~s}, 3 \mathrm{H}), 3.79(\mathrm{~s}, 2 \mathrm{H}), 6.60(\mathrm{~d}, J=$ $10.4 \mathrm{~Hz}, 1 \mathrm{H}), 7.18-7.26$ (m, 3H), 739-7.41 (m, 2H); ${ }^{13} \mathrm{C}$ NMR (100 $\left.\mathrm{MHz}, \mathrm{CDCl}_{3}\right): \delta 19.1,22.1,28.4,31.4,52.0,125.9,127.1,128.8$, 128.9, 131.9, 135.9, 152.0, 167.4; HRMS (ESI) exact mass calcd for $\mathrm{C}_{14} \mathrm{H}_{18} \mathrm{O}_{2} \mathrm{~S}+\mathrm{Na}(\mathrm{M}+\mathrm{Na}), 273.0925$; found: 273.0920 .
Methyl-(E)-2-(((4-chlorophenyl)thio)methyl)-4-methylpent-2enoate (4x). The title compound was prepared following the general procedure for Table 2, using allyl iodide 1d i.e. methyl(E)-2-(iodomethyl)-4-methylpent-2-enoate (2.0 mmol, $0.536 \mathrm{~g}$ ), bis(3-chlorophenyl)disulfide $(2.0 \mathrm{mmol}, 0.574 \mathrm{~g}), \quad$ DTBP (10 mmol, $1.46 \mathrm{~g}, 1.8 \mathrm{~mL}$ ) and $\mathrm{CH}_{3} \mathrm{CN}(2.0 \mathrm{~mL})$, providing $4 \mathbf{x}$ as pale yellow liquid. Yield: $0.398 \mathrm{~g}, 70 \%$; ${ }^{1} \mathrm{H}$ NMR $(400 \mathrm{MHz}$, $\left.\mathrm{CDCl}_{3}\right): \delta 0.87(\mathrm{~s}, 3 \mathrm{H}), 0.88(\mathrm{~s}, 3 \mathrm{H}), 2.39-2.45(\mathrm{~m}, 1 \mathrm{H}), 3.72(\mathrm{~s}$, $3 \mathrm{H}), 3.76(\mathrm{~s}, 2 \mathrm{H}), 6.62(\mathrm{~d}, J=10.4 \mathrm{~Hz}, 1 \mathrm{H}), 7.22(\mathrm{dd}, J=8.4 \mathrm{~Hz} \&$ $2.0 \mathrm{~Hz}, 2 \mathrm{H}), 7.32(\mathrm{dd}, J=8.4 \mathrm{~Hz} \& 2.0 \mathrm{~Hz}, 2 \mathrm{H}) ;{ }^{13} \mathrm{C}$ NMR (100 $\left.\mathrm{MHz} \mathrm{CDCl}_{3}\right): \delta 22.1,28.4,31.6,52.0,125.6,128.9,133.2,134.4$, 152.3, 167.2; HRMS (ESI) exact mass calcd for $\mathrm{C}_{14} \mathrm{H}_{17} \mathrm{ClO}_{2} \mathrm{~S}+\mathrm{Na}$ $(\mathrm{M}+\mathrm{Na}), 307.0535$; found: 307.0539 .

\section{General procedure for Table 3}

To a stirred solution of allyl bromide $5(2.0 \mathrm{mmol})$ and disulfide $(2.0 \mathrm{mmol})$ in $\mathrm{CH}_{3} \mathrm{CN}(2.0 \mathrm{~mL})$ was added DTBP $(10.0 \mathrm{mmol})$, then the reaction mixture was stirred for $48 \mathrm{~h}$ at $80^{\circ} \mathrm{C}$ under nitrogen atmosphere. The solvent was then removed under reduced pressure and the crude product thus obtained was purified by column chromatography (silica gel, 1\% EtOAc in hexanes) to provide the allyl thioether 6.

(E)-3-Phenyl-2-((phenylthio)methyl)acrylonitrile (6a). ${ }^{10 c}$ The title compound was prepared following the general procedure for Table 3, using allyl iodide 5a i.e. (E)-2-(iodomethyl)-3phenylacrylonitrile $(2.0 \mathrm{mmol}, 0.538 \mathrm{~g})$, diphenyl disulfide (2.0 mmol, $0.436 \mathrm{~g}$ ), DTBP (10 mmol, $1.46 \mathrm{~g}, 1.8 \mathrm{~mL})$ and $\mathrm{CH}_{3} \mathrm{CN}$ $(2.0 \mathrm{~mL})$, providing $6 \mathbf{a}$ as pale yellow liquid. Yield: $0.426 \mathrm{~g}, 85 \%$; ${ }^{1} \mathrm{H}$ NMR (400 MHz, $\mathrm{CDCl}_{3}$ ): $\delta 3.73(\mathrm{~s}, 2 \mathrm{H}), 6.65$ (s, 1H), 7.27-7.35

Table 3 DTBP-promoted C-S bond formation between allyl iodides 5 and disulfides $2^{a, b}$
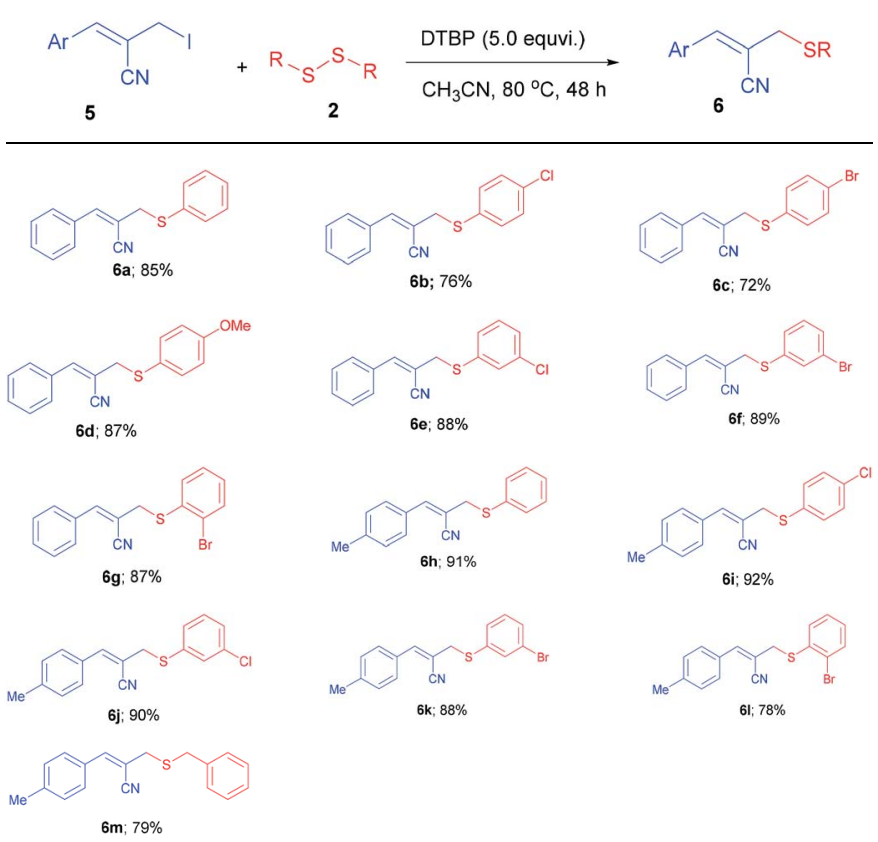

${ }^{a}$ Reaction conditions: allyl iodide $5(2.0 \mathrm{mmol})$, disulfide $2(2.0 \mathrm{mmol})$ and DTBP $(10.0 \mathrm{mmol})$ were reacted in $\mathrm{CH}_{3} \mathrm{CN}(2.0 \mathrm{~mL})$ at $80^{\circ} \mathrm{C}$ for $48 \mathrm{~h}$. ${ }^{b}$ Isolated yields are based on allyl iodide $\mathbf{5}$. 
(m, 6H), 7.44-7.46 (m, 2H), 7.58-7.59 (m, 2H); ${ }^{13} \mathrm{C}$ NMR (100 $\left.\mathrm{MHz}, \mathrm{CDCl}_{3}\right): \delta 41.1,107.7,118.2,128.1,128.8,128.9,129.3$, $130.5,132.9,133.1,133.5,144.9$.

(E)-2-(((4-Chlorophenyl)thio)methyl)-3-phenylacrylonitrile (6b). The title compound was prepared following the general procedure for Table 3, using allyl iodide 5a i.e. (E)-2-(iodomethyl)-3phenylacrylonitrile (2.0 mmol, $0.538 \mathrm{~g})$, bis(4-chlorophenyl) disulfide (2.0 mmol, $0.574 \mathrm{~g}$ ), DTBP (10 mmol, $1.46 \mathrm{~g}, 1.8 \mathrm{~mL}$ ) and $\mathrm{CH}_{3} \mathrm{CN}(2.0 \mathrm{~mL})$, providing $6 \mathbf{b}$ as pale yellow liquid. Yield: $0.433 \mathrm{~g}, 76 \% ;{ }^{1} \mathrm{H}$ NMR (400 MHz, $\left.\mathrm{CDCl}_{3}\right): \delta 3.71(\mathrm{~s}, 2 \mathrm{H}), 6.68(\mathrm{~s}$, $1 \mathrm{H}), 7.24(\mathrm{dd}, J=8.8 \mathrm{~Hz} \& 2.0 \mathrm{~Hz}, 2 \mathrm{H}), 7.35-7.37(\mathrm{~m}, 5 \mathrm{H}$; multiplet conations one doublet at $\delta 7.36, J=8.4 \mathrm{~Hz}, 2 \mathrm{H}$ and multiplet for $3 \mathrm{H}), 7.58-7.61(\mathrm{~m}, 2 \mathrm{H}) ;{ }^{13} \mathrm{C} \mathrm{NMR}(100 \mathrm{MHz}$, $\left.\mathrm{CDCl}_{3}\right): \delta 41.2,107.5,118.0,128.8,128.9,129.4,130.7,132.0$, 132.9, 134.2, 134.3, 145.1, HRMS (ESI) exact mass calcd for $\mathrm{C}_{16} \mathrm{H}_{12} \mathrm{ClNS}+\mathrm{Na}(\mathrm{M}+\mathrm{Na}), 308.0277$; found: 308.0092 .

(E)-2-((4-Bromophenyl)thio)methyl)-3-phenylacrylonitrile (6c). The title compound was prepared following the general procedure for Table 3, using allyl iodide 5a i.e. (E)-2-(iodomethyl)-3phenylacrylonitrile $(2.0 \mathrm{mmol}, 0.538 \mathrm{~g})$, bis(4-bromophenyl) disulfide (2.0 mmol, $0.752 \mathrm{~g}$ ), DTBP (10 mmol, $1.46 \mathrm{~g}, 1.8 \mathrm{~mL}$ ) and $\mathrm{CH}_{3} \mathrm{CN}(2.0 \mathrm{~mL})$, providing $6 \mathrm{c}$ as yellow solid. $\mathrm{Mp}: 83{ }^{\circ} \mathrm{C}$; yield: $0.475 \mathrm{~g}, 72 \% ;{ }^{1} \mathrm{H}$ NMR (400 $\mathrm{MHz}, \mathrm{CDCl}_{3}$ ): $\delta 3.72(\mathrm{~s}, 2 \mathrm{H})$, $6.70(\mathrm{~s}, 1 \mathrm{H}), 7.28(\mathrm{~d}, J=8.4 \mathrm{~Hz}, 2 \mathrm{H}), 7.36-7.40$ (m, 5H, multiplet contains one doublet at $\delta 7.37, J=8.4 \mathrm{~Hz}, 2 \mathrm{H}$ and one multiplet for $3 \mathrm{H}), 7.59-7.61(\mathrm{~m}, 2 \mathrm{H}) ;{ }^{13} \mathrm{C} \mathrm{NMR}\left(100 \mathrm{MHz}, \mathrm{CDCl}_{3}\right): \delta 41.0$, $107.4,118.0,122.3,128.8,129.0,130.7,132.3$, 132.7, 132.9, 134.3, 145.1; HRMS (ESI) exact mass calcd for $\mathrm{C}_{16} \mathrm{H}_{12} \mathrm{BrNS}+\mathrm{Na}$ $(\mathrm{M}+\mathrm{Na}), 351.9772$; found: 351.9749 .

(E)-2-(((4-Methoxyphenyl)thio)methyl)-3-phenylacrylonitrile (6d). The title compound was prepared following the general procedure for Table 3, using allyl iodide 5a i.e. (E)-2(iodomethyl)-3-phenylacrylonitrile $(2.0 \mathrm{mmol}, 0.538 \mathrm{~g})$, bis(4methoxyphenyl)disulfide $(2.0 \mathrm{mmol}, 0.556 \mathrm{~g})$, DTBP $(10 \mathrm{mmol}$, $1.46 \mathrm{~g}, 1.8 \mathrm{~mL})$ and $\mathrm{CH}_{3} \mathrm{CN}(2.0 \mathrm{~mL})$, providing $\mathbf{6 d}$ as pale yellow liquid. Yield: $0.488 \mathrm{~g}, 87 \% ;{ }^{1} \mathrm{H} \mathrm{NMR}\left(400 \mathrm{MHz} \mathrm{CDCl}_{3}\right): \delta 3.60$ (s, $2 \mathrm{H}), 3.71(\mathrm{~s}, 3 \mathrm{H}), 6.47$ (s, 1H), 6.79 (d, $J=8.8 \mathrm{~Hz}, 2 \mathrm{H}), 7.32-7.34$ $(\mathrm{m}, 3 \mathrm{H}), 7.38(\mathrm{~d}, J=8.8 \mathrm{~Hz}, 2 \mathrm{H}), 7.53-7.55(\mathrm{~m}, 2 \mathrm{H}) ;{ }^{13} \mathrm{C}$ NMR $\left(100 \mathrm{MHz}, \mathrm{CDCl}_{3}\right): \delta 42.7,55.4,108.0,114.8,118.2,123.5,128.7$, 128.9, 130.4, 133.1, 136.3, 144.7, 160.1; HRMS (ESI) exact mass calcd for $\mathrm{C}_{17} \mathrm{H}_{15} \mathrm{NOS}+\mathrm{K}(\mathrm{M}+\mathrm{K}), 320.0511$; found: 320.0517 .

(E)-2-(((3-Chlorophenyl)thio)methyl)-3-phenylacrylonitrile (6e). The title compound was prepared following the general procedure for Table 3, using allyl iodide 5a i.e. (E)-2-(iodomethyl)-3phenylacrylonitrile $(2.0 \mathrm{mmol}, 0.538 \mathrm{~g})$, bis(3-chlorophenyl) disulfide (2.0 mmol, $0.574 \mathrm{~g}$ ), DTBP (10 mmol, $1.46 \mathrm{~g}, 1.8 \mathrm{~mL}$ ) and $\mathrm{CH}_{3} \mathrm{CN}(2.0 \mathrm{~mL})$, providing $6 \mathrm{e}$ as pale yellow liquid. Yield: $0.502 \mathrm{~g}, 88 \% ;{ }^{1} \mathrm{H}$ NMR (400 MHz, $\left.\mathrm{CDCl}_{3}\right): \delta 3.75(\mathrm{~s}, 2 \mathrm{H}), 6.76(\mathrm{~s}$, 1H), 7.07-7.22 (m, 2H), 7.24-7.44 (m, 5H), 7.55-7.65 (m, 2H); ${ }^{13} \mathrm{C}$ NMR $\left(100 \mathrm{MHz}, \mathrm{CDCl}_{3}\right): \delta 40.5,107.2,118.0,128.0,128.9$, 129.0, 130.2, 130.3, 130.7, 131.7, 132.9, 134.8, 135.7, 145.3; HRMS (ESI) exact mass calcd for $\mathrm{C}_{16} \mathrm{H}_{12} \mathrm{ClNS}+\mathrm{Na}(\mathrm{M}+\mathrm{Na})$, 308.0277; found: 308.0091.

(E)-2-(((3-Bromophenyl)thio)methyl)-3-phenylacrylonitrile (6f). The title compound was prepared following the general procedure for Table 3, using allyl iodide 5a i.e. (E)-2-(iodomethyl)-3phenylacrylonitrile (2.0 mmol, $0.538 \mathrm{~g})$, bis(3-bromophenyl) disulfide (2.0 mmol, $0.752 \mathrm{~g}$ ), DTBP (10 mmol, $1.46 \mathrm{~g}, 1.8 \mathrm{~mL}$ ) and $\mathrm{CH}_{3} \mathrm{CN}(2.0 \mathrm{~mL})$, providing $\mathbf{6 f}$ as pale yellow liquid. Yield: $0.587 \mathrm{~g}, 89 \% ;{ }^{1} \mathrm{H}$ NMR $\left(400 \mathrm{MHz}, \mathrm{CDCl}_{3}\right): \delta 3.74(\mathrm{~s}, 2 \mathrm{H}), 6.76(\mathrm{~s}$, $1 \mathrm{H}), 7.11(\mathrm{t}, J=8.0 \mathrm{~Hz}, 1 \mathrm{H}), 7.21-7.41(\mathrm{~m}, 5 \mathrm{H}), 7.56(\mathrm{~s}, 1 \mathrm{H}), 7.61-$ $7.66(\mathrm{~m}, 2 \mathrm{H}) ;{ }^{13} \mathrm{C}$ NMR $\left(100 \mathrm{MHz}, \mathrm{CDCl}_{3}\right): \delta 40.6,107.2,118.0$, 122.9, 128.9, 129.05, 129.06, 130.7, 130.8, 130.9, 132.9, 134.5, 136.1 145.4; HRMS (ESI) exact mass calcd for $\mathrm{C}_{16} \mathrm{H}_{12} \mathrm{BrNS}+\mathrm{Na}$ $(\mathrm{M}+\mathrm{Na}), 351.9772$; found: 351.9625 .

(E)-2-(((2-Bromophenyl)thio)methyl)-3-phenylacrylonitrile (6g). The title compound was prepared following the general procedure for Table 3, using allyl iodide 5a i.e. (E)-2-(iodomethyl)-3phenylacrylonitrile (2.0 mmol, $0.538 \mathrm{~g})$, bis(2-bromophenyl) disulfide (2.0 mmol, $0.752 \mathrm{~g}$ ), DTBP (10 mmol, $1.46 \mathrm{~g}, 1.8 \mathrm{~mL}$ ) and $\mathrm{CH}_{3} \mathrm{CN}(2.0 \mathrm{~mL})$, providing $6 \mathrm{~g}$ as yellow solid. Mp: $79{ }^{\circ} \mathrm{C}$; yield: $0.574 \mathrm{~g}, 87 \%$; ${ }^{1} \mathrm{H}$ NMR $\left(400 \mathrm{MHz}, \mathrm{CDCl}_{3}\right): \delta 3.81(\mathrm{~s}, 2 \mathrm{H})$, $6.74(\mathrm{~s}, 1 \mathrm{H}), 7.10(\mathrm{td}, J=7.6 \mathrm{~Hz} \& 1.6 \mathrm{~Hz}, 1 \mathrm{H}), 7.21(\mathrm{td}, J=7.6 \mathrm{~Hz}$ \& $1.2 \mathrm{~Hz}, 1 \mathrm{H}), 7.31-7.37(\mathrm{~m}, 3 \mathrm{H}), 7.45(\mathrm{dd}, J=8.0 \mathrm{~Hz} \& 1.6 \mathrm{~Hz}$, 2H), 7.53-7.62 (m, 3H); ${ }^{13} \mathrm{C}$ NMR (100 MHz, $\left.\mathrm{CDCl}_{3}\right): \delta 39.5$, $106.8,118.1$, 127.3, 128.2, 128.8, 128.9, 129.3, 130.7, 133.0, 133.5, 133.6, 134.5, 145.4; HRMS (ESI) exact mass calcd for $\mathrm{C}_{16} \mathrm{H}_{12} \mathrm{BrNS}+\mathrm{Na}(\mathrm{M}+\mathrm{Na}), 351.9772$; found: 351.9713 .

(E)-2-((Phenylthio)methyl)-3-(p-tolyl)acrylonitrile (6h). ${ }^{10 c}$ The title compound was prepared following the general procedure for Table 3, using allyl iodide $5 \mathbf{b}$ i.e. (E)-2-(iodomethyl)-3-(p-tolyl) acrylonitrile (2.0 mmol, $0.566 \mathrm{~g})$, diphenyl disulfide $(2.0 \mathrm{mmol}$, $0436 \mathrm{~g}$ ), DTBP (10 mmol, $1.46 \mathrm{~g}, 1.8 \mathrm{~mL}$ ) and $\mathrm{CH}_{3} \mathrm{CN}(2.0 \mathrm{~mL}$ ), providing $6 \mathbf{h}$ as pale yellow liquid. Yield: $0.482 \mathrm{~g}, 91 \% ;{ }^{1} \mathrm{H}$ NMR $\left(400 \mathrm{MHz}, \mathrm{CDCl}_{3}\right): \delta 2.33(\mathrm{~s}, 3 \mathrm{H}), 3.71(\mathrm{~s}, 2 \mathrm{H}), 6.63(\mathrm{~s}, 1 \mathrm{H}), 7.14$ $(\mathrm{d}, J=8.0 \mathrm{~Hz}, 2 \mathrm{H}), 7.26-7.29(\mathrm{~m}, 3 \mathrm{H}), 7.44(\mathrm{~d}, J=8.0 \mathrm{~Hz}, 2 \mathrm{H})$, $7.52(\mathrm{~d}, J=8.0 \mathrm{~Hz}, 2 \mathrm{H}) ;{ }^{13} \mathrm{C} \mathrm{NMR}\left(100 \mathrm{MHz}, \mathrm{CDCl}_{3}\right): \delta 21.6,41.1$, 106.3 , 118.4, 128.0, 128.9, 129.3, 129.6, 130.4, 132.8, 133.7, 141.0, 144.9.

(E)-2-(((4-Chlorophenyl)thio)methyl)-3-(p-tolyl)acrylonitrile (6i). The title compound was prepared following the general procedure for Table 3, using allyl iodide $5 \mathbf{b}$ i.e. (E)-2-(iodomethyl)-3(p-tolyl)acrylonitrile $(2.0 \mathrm{mmol}, 0.566 \mathrm{~g})$, bis(4-chlorophenyl) disulfide (2.0 mmol, $0.574 \mathrm{~g}$ ), DTBP (10 mmol, $1.46 \mathrm{~g}, 1.8 \mathrm{~mL}$ ) and $\mathrm{CH}_{3} \mathrm{CN}(2.0 \mathrm{~mL})$, providing $6 \mathbf{i}$ as yellow solid. $\mathrm{Mp}: 73{ }^{\circ} \mathrm{C}$; yield: $0.551 \mathrm{~g}, 92 \% ;{ }^{1} \mathrm{H}$ NMR $\left(400 \mathrm{MHz}, \mathrm{CDCl}_{3}\right): \delta 2.35(\mathrm{~s}, 3 \mathrm{H})$, $3.71(\mathrm{~s}, 2 \mathrm{H}), 6.65(\mathrm{~s}, 1 \mathrm{H}), 7.17(\mathrm{~d}, J=8.0 \mathrm{~Hz}, 2 \mathrm{H}), 7.24(\mathrm{~d}, J=$ $8.4 \mathrm{~Hz}, 2 \mathrm{H}), 7.36(\mathrm{~d}, J=8.8 \mathrm{~Hz}, 2 \mathrm{H}), 7.52(\mathrm{~d}, J=8.4 \mathrm{~Hz}, 2 \mathrm{H}) ;{ }^{13} \mathrm{C}$ NMR (100 MHz, $\left.\mathrm{CDCl}_{3}\right): \delta 22.7,41.3,106.1,118.1,128.8,129.3$, 129.6, 130.2, 132.1, 134.20, 134.29, 141.2, 145.0; HRMS (ESI) exact mass calcd for $\mathrm{C}_{17} \mathrm{H}_{14} \mathrm{ClNS}+\mathrm{K}(\mathrm{M}+\mathrm{K})$, 338.0173; found: 338.0179 .

(E)-2-(((3-Chlorophenyl)thio)methyl)-3-(p-tolyl)acrylonitrile (6j). The title compound was prepared following the general procedure for Table 3, using allyl iodide $5 \mathbf{b}$ i.e. (E)-2-(iodomethyl)-3(p-tolyl)acrylonitrile (2.0 mmol, $0.566 \mathrm{~g})$, bis(3-chlorophenyl) disulfide (2.0 mmol, $0.574 \mathrm{~g}$ ), DTBP (10 mmol, $1.46 \mathrm{~g}, 1.8 \mathrm{~mL}$ ) and $\mathrm{CH}_{3} \mathrm{CN}(2.0 \mathrm{~mL})$, providing $\mathbf{6 j}$ as yellow solid. $\mathrm{Mp}: 70{ }^{\circ} \mathrm{C}$; yield: $0.539 \mathrm{~g}, 90 \% ;{ }^{1} \mathrm{H}$ NMR $\left(400 \mathrm{MHz}, \mathrm{CDCl}_{3}\right): \delta 2.31(\mathrm{~s}, 3 \mathrm{H})$, $3.74(\mathrm{~s}, 2 \mathrm{H}), 6.75(\mathrm{~s}, 1 \mathrm{H}), 7.13(\mathrm{~d}, J=7.6 \mathrm{~Hz}, 2 \mathrm{H}), 7.16-7.17(\mathrm{~m}$, 2H), 7.22-7.31 (m, 1H), 7.39 (s, 1H), 7.46-7.56 (m, 2H); ${ }^{13} \mathrm{C}$ NMR $\left(100 \mathrm{MHz}, \mathrm{CDCl}_{3}\right): \delta 21.6,40.5,105.8,118.3,127.8,128.9,129.7$, 130.0, 130.2 130.3, 131.4, 134.7, 136.0, 141.2, 145.4; HRMS (ESI) 
exact mass calcd for $\mathrm{C}_{17} \mathrm{H}_{14} \mathrm{ClNS}+\mathrm{Na}(\mathrm{M}+\mathrm{Na}), 322.0433$; found: 322.0439 .

(E)-2-(((3-Bromophenyl)thio)methyl)-3-(p-tolyl)acrylonitrile (6k). The title compound was prepared following the general procedure for Table 3, using allyl iodide 5b i.e. (E)-2-(iodomethyl)-3-(p-tolyl)acrylonitrile $(2.0 \mathrm{mmol}, \quad 0.566 \mathrm{~g}), \quad$ bis(3bromophenyl)disulfide (2.0 mmol, $0.752 \mathrm{~g})$, DTBP $(10 \mathrm{mmol}$, $1.46 \mathrm{~g}, 1.8 \mathrm{~mL}$ ) and $\mathrm{CH}_{3} \mathrm{CN}(2.0 \mathrm{~mL})$, providing 6k as yellow solid. Mp: $79{ }^{\circ} \mathrm{C}$; yield: $0.605 \mathrm{~g}, 88 \% ;{ }^{1} \mathrm{H}$ NMR (400 MHz, $\mathrm{CDCl}_{3}$ ): $\delta 2.35(\mathrm{~s}, 3 \mathrm{H}), 3.75(\mathrm{~s}, 2 \mathrm{H}), 6.73(\mathrm{~s}, 1 \mathrm{H}), 7.10-7.20(\mathrm{~m}, 3 \mathrm{H}), 7.30-$ 7.39 (m, 2H), 7.52-7.58 (m, 3H); ${ }^{13} \mathrm{C} \mathrm{NMR} \mathrm{(100} \mathrm{MHz,} \mathrm{CDCl}_{3}$ ): $\delta 21.6,40.7,105.8,118.2,122.8,128.9,129.7,130.1,130.5,130.7$, 130.8, 134.5, 136.1, 141.3, 145.4; HRMS (ESI) exact mass calcd for $\mathrm{C}_{17} \mathrm{H}_{14} \mathrm{BrNS}+\mathrm{K}(\mathrm{M}+\mathrm{K}), 381.9667$; found: 381.9666 .

(E)-2-((2-Bromophenyl)thio)methyl)-3-(p-tolyl)acrylonitrile (61). The title compound was prepared following the general procedure for Table 3, using allyl iodide $5 \mathbf{b}$ i.e. (E)-2-(iodomethyl)-3-( $p$-tolyl)acrylonitrile $(2.0 \mathrm{mmol}, 0.566 \mathrm{~g}), \quad \operatorname{bis}(2-$ bromophenyl)disulfide (2.0 mmol, $0.752 \mathrm{~g})$, DTBP $(10 \mathrm{mmol}$, $1.46 \mathrm{~g}, 1.8 \mathrm{~mL}$ ) and $\mathrm{CH}_{3} \mathrm{CN}(2.0 \mathrm{~mL})$, providing 61 as yellow solid. Mp: $69{ }^{\circ} \mathrm{C}$; yield: $0.537 \mathrm{~g}, 78 \% ;{ }^{1} \mathrm{H}$ NMR $\left(400 \mathrm{MHz}, \mathrm{CDCl}_{3}\right): \delta 2.34$ (s, 3H), $3.80(\mathrm{~s}, 2 \mathrm{H}), 6.71(\mathrm{~s}, 1 \mathrm{H}), 7.10(\mathrm{td}, J=7.6 \mathrm{~Hz} \& 1.6 \mathrm{~Hz}$, $1 \mathrm{H}), 7.15$ (d, $J=8.0 \mathrm{~Hz}, 2 \mathrm{H}), 7.21(\mathrm{td}, J=7.6 \mathrm{~Hz}, \& 1.2 \mathrm{~Hz}, 1 \mathrm{H})$, $7.43(\mathrm{dd}, J=8.0 \mathrm{~Hz} \& 1.6 \mathrm{~Hz}, 1 \mathrm{H}), 7.49$ (d, $J=8.0 \mathrm{~Hz}, 2 \mathrm{H}), 7.58$ $(\mathrm{dd}, J=8.0 \mathrm{~Hz} \& 1.6 \mathrm{~Hz}, 1 \mathrm{H}) ;{ }^{13} \mathrm{C} \mathrm{NMR}\left(100 \mathrm{MHz}, \mathrm{CDCl}_{3}\right): \delta 21.6$, 39.5, 105.5, 118.3, 127.5, 128.1, 128.8, 129.3, 129.6, 130.2, 133.4, 133.9, 141.2, 145.4; HRMS (ESI) exact mass calcd for $\mathrm{C}_{17} \mathrm{H}_{14} \mathrm{BrNS}+\mathrm{K}(\mathrm{M}+\mathrm{K}), 381.9667$; found: 381.9673 .

(E)-2-((benzylthio)methyl)-3-(p-tolyl)acrylonitrile (6m). The title compound was prepared following the general procedure for Table 3, using allyl iodide $5 \mathbf{b}$ i.e. (E)-2-(iodomethyl)-3-( $p$-tolyl) acrylonitrile (2.0 mmol, $0.566 \mathrm{~g})$, dibenzyl disulfide $(2.0 \mathrm{mmol}$, $0.492 \mathrm{~g}$ ), DTBP (10 mmol, $1.46 \mathrm{~g}, 1.8 \mathrm{~mL}$ ) and $\mathrm{CH}_{3} \mathrm{CN}(2.0 \mathrm{~mL})$, providing $6 \mathrm{~m}$ as yellow liquid; yield: $0.441 \mathrm{~g}, 79 \% ;{ }^{1} \mathrm{H}$ NMR $(400$ $\left.\mathrm{MHz} \mathrm{CDCl}_{3}\right): \delta 2.40(\mathrm{~s}, 3 \mathrm{H}), 3.31(\mathrm{~s}, 2 \mathrm{H}), 3.76(\mathrm{~s}, 2 \mathrm{H}), 6.83(\mathrm{~s}$, $1 \mathrm{H}), 7.24(\mathrm{~d}, J=8.0 \mathrm{~Hz}, 2 \mathrm{H}), 7.31-7.35(\mathrm{~m}, 5 \mathrm{H}), 7.67(\mathrm{~d}, J=$ $8.0 \mathrm{~Hz}, 2 \mathrm{H}) ;{ }^{13} \mathrm{C} \mathrm{NMR}\left(100 \mathrm{MHz}, \mathrm{CDCl}_{3}\right): \delta 21.6,35.4,36.2,106.7$, $118.5,127.4,128.8,129.0,129.1,129.7,130.3,137.2,141.2$, 144.6; HRMS (ESI) exact mass calcd for $\mathrm{C}_{18} \mathrm{H}_{17} \mathrm{NS}+\mathrm{K}(\mathrm{M}+\mathrm{K})$, 318.0719; found: 318.0726.

Methyl (E)-3-phenyl-2-(((2,2,6,6-tetramethylpiperidin-1-yl) oxy)methyl)acrylate (7). To a stirred solution of allyl iodide 1a i.e. methyl-(Z)-2-(iodomethyl)-3-phenylacrylate (2.0 mmol, 0.604 $\mathrm{g}$ ) in $\mathrm{CH}_{3} \mathrm{CN}(2.0 \mathrm{~mL})$ was added TEMPO (2.0 mmol, $0.312 \mathrm{~g}$ ) and then the reaction mixture was stirred for $80{ }^{\circ} \mathrm{C}$ under nitrogen atmosphere for $48 \mathrm{~h}$. The solvent was then removed under reduced pressure and the crude product thus obtained was purified by column chromatography (silica gel, 1\% EtOAc in hexanes) to provide the allyl thioether 7 as pale yellow colour liquid. Yield: $0.543 \mathrm{~g}, 82 \% ;{ }^{1} \mathrm{H} \mathrm{NMR}\left(400 \mathrm{MHz}, \mathrm{CDCl}_{3}\right): \delta 1.06$ (s, $6 \mathrm{H}), 1.09(\mathrm{~s}, 6 \mathrm{H}), 1.40-1.42(\mathrm{~m}, 6 \mathrm{H}), 3.82(\mathrm{~s}, 3 \mathrm{H}), 4.67(\mathrm{~s}, 2 \mathrm{H})$, 7.34-7.68 (m, 3H), 7.47-7.49 (m, 2H), $7.83(\mathrm{~s}, 1 \mathrm{H}) ;{ }^{13} \mathrm{C}$ NMR $(100$ $\left.\mathrm{MHz}, \mathrm{CDCl}_{3}\right): \delta 17.1,20.2,32.9,40.0,52.0,59.9,70.8,128.3$, 128.9, 129.5, 135.1, 143.6, 168.5; HRMS (ESI) exact mass calcd for $\mathrm{C}_{20} \mathrm{H}_{29} \mathrm{NO}_{3}+\mathrm{K}(\mathrm{M}+\mathrm{K}), 370.1785$; found: 370.1778 .

\section{Acknowledgements}

SERB-DST, New Delhi (YSS/2015/001870) and UGC-India (Startup grant) are gratefully acknowledged for financial support. P. S. and R. C. thanks the UGC and R. B. thanks the SERB-DST for their fellowships. S. S. B. is DST INSPIRE Faculty (IFA-2014/CH167), DST-India. We thank MRC-MNIT Jaipur for NMR and USIC University of Rajasthan, Jaipur for HRMS \& GCMS data collection.

\section{Notes and references}

1 (a) C.-L. Sun and Z.-J. Shi, Chem. Rev., 2014, 114, 9219 and reference cited therein; $(b)$ C. R. Reddy and M. D. Reddy, J. Org. Chem., 2014, 79, 106; (c) M. Ochiai, Y. Takeuchi, T. Katayama, T. Sueda and K. Miyamoto, J. Am. Chem. Soc., 2005, 127, 12244; (d) T. Dohi, A. Maruyama, M. Yoshimura, K. Morimoto, H. Tohma and Y. Kita, Angew. Chem., Int. Ed., 2005, 44, 6193; (e) M. Uyanik, D. Suzuki, T. Yasui and K. Ishihara, Angew. Chem., Int. Ed., 2011, 50, 5331; (f) S. K. R. Parumala and R. K. Peddinti, Green Chem., 2015, 17, 4068; (g) Y. Siddaraju and K. R. Prabhu, J. Org. Chem., 2016, 81, 7838.

2 (a) R. N. Reddi, P. K. Prasad and A. Sudalai, Org. Lett., 2014, 16, 5674; (b) J. Huang, L. Li, H. Li, E. Husan, P. Wang and B. Wang, Chem. Commun., 2012, 48, 10204; (c) G. Songjin, Y. Jin-Tao, D. Qiang, Y. Haitao and C. Jiang, Chem. Commun., 2014, 50, 6240; (d) A. Wagner and A. R. Ofial, J. Org. Chem., 2015, 80, 2848; (e) J. Meesin, P. Katrun, C. Pareseecharoen, M. Pohmakotr, V. Reutrakul, D. Soorukram and C. Kuhakarn, J. Org. Chem., 2016, 81, 2744 .

3 (a) S. V. Ley and A. W. Thomas, Angew. Chem., Int. Ed., 2003, 42, 5400; (b) T. Kondo and T.-a. Mitsudo, Chem. Rev., 2000, 100, 3205; (c) I. P. Beletskaya and V. P. Ananikov, Chem. Rev., 2011, 111, 1596; (d) A. Gangjee, Y. Zeng, T. Talreja, J. J. McGuire, R. L. Kisliuk and S. F. Queener, J. Med. Chem., 2007, 50, 3046; (e) G. Liu, J. R. Huth, E. T. Olejniczak, F. Mendoza, S. W. Fesik and T. W. von Geldern, J. Med. Chem., 2001, 44, 1202; $(f)$ G. De Martino, G. La Regina, A. Coluccia, M. C. Edler, M. C. Barbera, A. Brancale, E. Wilcox, E. Hamel, M. Artico and R. Silvestri, J. Med. Chem., 2004, 47, 6120; (g) J. Hutton, A. D. Jones, S. A. Lee, D. M. G. Martin, B. R. Meyrick, I. Patel, R. F. Peardon and L. Powell, Org. Process Res. Dev., 1997, 1, 61; (h) S. W. Kaldor, V. J. Kalish, J. F. Davies, B. V. Shetty, J. E. Fritz, K. Appelt, J. A. Burgess, K. M. Campanale, N. Y. Chirgadze, D. K. Clawson, B. A. Dressman, S. D. Hatch, D. A. Khalil, M. B. Kosa, P. P. Lubbehusen, M. A. Muesing, A. K. Patick, S. H. Reich, K. S. Su and J. H. Tatlock, J. Med. Chem., 1997, 40, 3979; (i) A. Dondoni, Angew. Chem., Int. Ed., 2008, 47, 8995; (j) A. B. Lowe, Polym. Chem., 2010, 1, 17; (k) J. Liu, J. Yang, Q. Yang, G. Wang and Y. Li, Adv. Funct. Mater., 2005, 15, 1297.

4 (a) M.-L. Alcaraz, S. Atkinson, P. Cornwall, A. C. Foster, D. M. Gill, L. A. Humphries, P. S. Keegan, R. Kemp, E. Merifield, R. A. Nixon, A. J. Noble, D. O'Beirne, 
Z. M. Patel, J. Perkins, P. Rowan, P. Sadler, J. T. Singleton, J. Tornos, A. J. Watts and I. A. Woodland, Org. Process Res. Dev., 2005, 9, 555; (b) J. C. Sheehan and K. R. HeneryLogan, J. Am. Chem. Soc., 1959, 81, 3089; (c) S. Raghavan and B. Sridhar, J. Org. Chem., 2010, 75, 498; (d) C. Bharathi, K. J. Prabahar, C. S. Prasad, M. S. Rao, G. N. Trinadhachary, V. K. Handa, R. Dandala and A. Naidu, Pharmazie, 2008, 63, 14.

5 (a) T. Migita, T. Shimizu, Y. Asami, J. Shiobara, Y. Kato and M. Kosugi, Bull. Chem. Soc. Jpn., 1980, 53, 1385; (b) M. Kosugi, T. Ogata, M. Terada, H. Sano and T. Migita, Bull. Chem. Soc. Jpn., 1985, 58, 3657; (c) C. S. Bryan, J. A. Braunger and M. Lautens, Angew. Chem., Int. Ed., 2009, 48, 7064; (d) M. Kuhn, F. C. Falk and J. Paradies, Org. Lett., 2011, 13, 4100; (e) Y.-J. Chen and H. H. Chen, Org. Lett., 2006, 8, 5609; (f) J.-H. Cheng, C.-L. Yi, T.-J. Liu and C.-F. Lee, Chem. Commun., 2012, 48, 8440; $(g)$ Y.-A. Chen, S. S. Badsara, W.-T. Tsai and C.-F. Lee, Synthesis, 2015, 47, 181; (h) J.-R. Wu, C.-H. Lin and C.-F. Lee, Chem. Commun., 2009, 4450; (i) A. Correa, M. Carril and C. Bolm, Angew. Chem., Int. Ed., 2008, 47, 2880; (j) V. Percec, J.-Y. Bae and D. H. Hill, J. Org. Chem., 1995, 60, 6895; (k) Y. Zhang, K. C. Ngeow and J. Y. Ying, Org. Lett., 2007, 9, 3495; (l) V. P. Reddy, A. V. Kumar, K. Swapna and K. R. Rao, Org. Lett., 2009, 11, 1697; $(m)$ Y.-C. Wong, T. T. Jayanth and C.-H. Cheng, Org. Lett., 2006, 8, 5613; (n) N. Morita and N. Krause, Angew. Chem., Int. Ed., 2006, 45, 1897; (o) R. Das and D. Chakraborty, Tetrahedron Lett., 2012, 53, 7023; $(p)$ T.-J. Liu, C.-L. Yi, C.-C. Chan and C.-F. Lee, Chem.-Asian J., 2013, 8, 1029.

6 (a) C.-F. Lee, Y.-C. Liu and S. S. Badsara, Chem.-Asian J., 2014, 9, 706; (b) D. J. Procter, J. Chem. Soc., Perkin Trans. 1, 2001, 335; (c) H. Liu and X. Jiang, Chem.-Asian J., 2013, 8, 2546.

7 (a) J. Feng, G. Lu, M. Lv and C. Cai, Synlett, 2015, 26, 915; (b) J. Feng, M.-F. Lv, G.-P. Lu and C. Cai, Org. Biomol. Chem., 2015, 13, 677; (c) S.-r. Guo, Y.-q. Yuan and J.-n. Xiang, Org. Lett., 2013, 15, 4654; (d) B. Du, B. Jin and P. Sun, Org. Lett., 2014, 16, 3032; (e) J.-W. Zeng, Y.-C. Liu, P.-A. Hsieh, Y.-T. Huang, C.-L. Yi, S. S. Badsara and C.-F. Lee, Green Chem., 2014, 16, 2644; (f) S. S. Badsara, Y.-C. Liu, P.-A. Hsieh, J.-W. Zeng, S.-Y. Lu, Y.-W. Liu and C.-F. Lee, Chem. Commun., 2014, 50, 11374; (g) J. Yuan, X. Ma, H. Yi, C. Liu and A. Lei, Chem. Commun., 2014, 50, 14386; $(h)$ R.-Y. Tang, Y.-X. Xie, Y.-L. Xie, J.-N. Xiang and J.-H. Li,
Chem. Commun., 2011, 47, 12867; (i) X. Zhu, X. Xie, P. Li, J. Guo and L. Wang, Org. Lett., 2016, 18, 1546; (j) B. V. Varun and K. R. Prabhu, J. Org. Chem., 2014, 79, 9655; (k) Y.-W. Liu, S. S. Badsara, Y.-C. Liu and C.-F. Lee, $R S C$ $A d v$., 2015, 5, 44299; (l) C. D. Prasad, S. J. Balkrishna, A. Kumar, B. S. Bhakuni, K. Shrimali, S. Biswas and S. Kumar, J. Org. Chem., 2013, 78, 1434.

8 (a) Z. Yang, W.-J. Hao, S.-L. Wang, J.-P. Zhang, B. Jiang, G. Li and S.-J. Tu, J. Org. Chem., 2015, 80, 9224; (b) Y. He, J. Li, S. Luo, J. Huang and Q. Zhu, Chem. Commun., 2016, 52, 8444; (c) P.-A. Hsieh, S. S. Badsara, C.-H. Tsai, D. M. Reddy and C.-F. Lee, Synlett, 2016, 27, 1557.

9 (a) K. Ajiki, M. Hirano and K. Tanaka, Org. Lett., 2005, 7, 4193; (b) B. C. Ranu and T. Mandal, J. Org. Chem., 2004, 69, 5793; (c) S. Chowdhury and S. Roy, Tetrahedron Lett., 1997, 38, 2149; (d) Y. Yatsumonji, Y. Ishida, A. Tsubouchi and T. Takeda, Org. Lett., 2007, 9, 4603; (e) P. S. Reddy, M. A. Reddy, B. Sreedhar and M. V. B. Rao, Synth. Commun., 2010, 40, 2075.

10 (a) D. Basavaiah, K. R. Reddy and N. Kumaragurubaran, Nat. Protoc., 2007, 2, 2665; (b) D. Basavaiah, B. S. Reddy and S. S. Badsara, Chem. Rev., 2010, 110, 5447; (c) K. Karnakar, K. Ramesh, S. N. Murty and Y. P. D. Nageswar, Helv. Chim. Acta, 2013, 96, 2276.

11 (a) A. Gruiec and A. Foucaud, New J. Chem., 1991, 15, 943; (b) D. Basavaiah, P. K. S. Sarma and A. K. D. Bhavani, J. Chem. Soc., Chem. Commun., 1994, 1091; (c) D. Basavaiah and P. K. S. Sarma, J. Chem. Soc., Chem. Commun., 1992, 955; (d) D. Basavaiah, A. K. D. Bhavani, S. Pandiaraju and P. K. S. Sarma, Synlett, 1995, 2434; (e) D. Basavaiah and S. Pandiaraju, Tetrahedron, 1996, 52, 2261; $(f)$ H. J. Lee, M. R. Seong and J. N. Kim, Tetrahedron Lett., 1998, 39, 6223; $(g)$ G. W. Kabalka, B. Venkataiah and G. Dong, Org. Lett., 2003, 5, 3803; (h) G. W. Kabalka, G. Dong, B. Venkataiah and C. Chen, J. Org. Chem., 2005, 70, 9207; (i) B. C. Ranu, K. Chattopadhyay and R. Jana, Tetrahedron Lett., 2007, 48, 3847; (j) M. L. Kantam, K. B. S. Kumar and B. Sreedhar, J. Org. Chem., 2008, 73, 320; (k) D. Basavaiah, S. S. Badsara and B. C. Sahu, Chem.-Eur. J., 2013, 19, 2961.

12 The allyl iodide 1d was obtained as E-isomer. Accordingly, the corresponding allylic thioethers $4 \mathbf{w}$ and $4 \mathbf{x}$ were also obtained as $E$-isomer.

13 P. O. Deane, J. J. Guthrie-Strachan, P. T. Kaye and R. E. Whittaker, Synth. Commun., 1998, 28, 2601. 